\title{
Traveling Waves for a Discrete Diffusion Epidemic Model with Delay
}

\author{
Jingdong Wei*, Zaili Zhen, Jiangbo Zhou and Lixin Tian
}

\begin{abstract}
This paper is concerned with traveling wave solutions in a discrete diffusion epidemic model with delayed transmission. Employing the way of contradictory discussions and the bilateral Laplace transform, we obtain the nonexistence of nontrivial positive bounded traveling wave solutions. Utilizing the super-/sub-solutions method and the fixed point theory, we derive the existence of nontrivial positive traveling wave solutions with both super-critical and critical speeds. Our results indicate that the critical speed is the minimal speed.
\end{abstract}

\section{Introduction}

Considering the environment which individuals live in can be divided into countably discrete niches and the influence of latent period of the disease, we investigate a discrete diffusion epidemic model with delay

$$
\left\{\begin{array}{l}
\frac{d S_{n}(t)}{d t}=d_{s}\left[S_{n+1}(t)+S_{n-1}(t)-2 S_{n}(t)\right]-\frac{\beta S_{n}(t) I_{n}(t-\tau)}{S_{n}(t)+I_{n}(t-\tau)+R_{n}(t)}, \\
\frac{d I_{n}(t)}{d t}=d_{i}\left[I_{n+1}(t)+I_{n-1}(t)-2 I_{n}(t)\right]+\frac{\beta S_{n}(t) I_{n}(t-\tau)}{S_{n}(t)+I_{n}(t-\tau)+R_{n}(t)}-(\gamma+\delta) I_{n}(t), \\
\frac{d R_{n}(t)}{d t}=d_{r}\left[R_{n+1}(t)+R_{n-1}(t)-2 R_{n}(t)\right]+\gamma I_{n}(t), \quad n \in \mathbb{Z},
\end{array}\right.
$$

where $S_{n}(t), I_{n}(t)$ and $R_{n}(t)$ refer to the densities of susceptible, infected and recovered individuals in time $t$ and niches $n$, respectively. The coefficients $d_{s}, d_{i}, d_{r}>0$ denote the diffusion rates of each class, $\beta>0$ stands for the transmission rate, $\gamma>0$ represents the recovery rate, $\delta \geq 0$ is the disease-induced death rate and $\tau \geq 0$ is the latent period. Model (1.1) with standard incidence $\beta S I /(S+I+R)$ describes that individuals can move freely in a patchy habitat and a part of infected individuals will be removed from the community due to disease-induced death, while other recovered individuals will return into the population, which capture the dynamical behavior of disease propagation.

In mathematical biology, traveling wave solutions can describe the phase transition that an epidemic transmits geographically with a constant speed from the initial state to

Received August 25, 2020; Accepted December 24, 2020.

Communicated by Je-Chiang Tsai.

2020 Mathematics Subject Classification. 34K10, 34C37, 92D25, 46N60.

Key words and phrases. discrete diffusion, epidemic model, latent period, super-/sub-solutions method, minimal speed.

${ }^{*}$ Corresponding author. 
the final state. The main purpose in this paper is to explore the existence and the minimal speed of traveling wave solutions for 1.1. . By a traveling wave solution with a constant speed $c$ of (1.1), we mean that it is in the form of

$$
\left(S_{n}, I_{n}, R_{n}\right)(t)=(S, I, R)(\xi), \quad \xi=n+c t
$$

which satisfies the following ordinary differential system

$$
\left\{\begin{array}{l}
c S^{\prime}(\xi)=d_{s}[S(\xi+1)+S(\xi-1)-2 S(\xi)]-\frac{\beta S(\xi) I(\xi-c \tau)}{S(\xi)+I(\xi-c \tau)+R(\xi)}, \\
c I^{\prime}(\xi)=d_{i}[I(\xi+1)+I(\xi-1)-2 I(\xi)]+\frac{\beta S(\xi) I(\xi-c \tau)}{S(\xi)+I(\xi-c \tau)+R(\xi)}-(\gamma+\delta) I(\xi), \\
c R^{\prime}(\xi)=d_{r}[R(\xi+1)+R(\xi-1)-2 R(\xi)]+\gamma I(\xi), \quad \xi \in \mathbb{R}
\end{array}\right.
$$

with the asymptotic boundary conditions

$$
(S, I, R)(-\infty)=\left(S_{1}, 0,0\right) \quad \text { and } \quad(S, I, R)(+\infty)=\left(S_{2}, 0, \gamma\left(S_{1}-S_{2}\right) /(\gamma+\delta)\right)
$$

where $S_{1}>0$ is a given constant and the constant $S_{2} \in\left[0, S_{1}\right)$ will be proved to exist.

In the last several decades, many theoretical issues concerning local-diffusion (or nonlocal-diffusion) epidemic models with (or without) time delay have been attracted considerable attention. Particularly, the existence and nonexistence of traveling waves for these models have been well-studied because these information can forecast whether or not an epidemic transmit in the crowd and how fast the epidemic invades geographically. In recent years, Wang et al. 20] studied a local-diffusion epidemic model

$$
\left\{\begin{array}{l}
\partial_{t} S=d_{1} \partial_{x x} S-\beta S I /(S+I+R) \\
\partial_{t} I=d_{2} \partial_{x x} I+\beta S I /(S+I+R)-(\gamma+\delta) I \\
\partial_{t} R=d_{3} \partial_{x x} R+\gamma I
\end{array}\right.
$$

where $S(x, t), I(x, t)$ and $R(x, t)$ are the densities of susceptible, infected and recovered individuals in location $x$ and time $t$, respectively. For the biological interpretation of model (1.3) and its coefficients, one can refer to 20]. They proved that if $R_{0}=\beta /(\gamma+\delta)>$ $1, c>c^{*}=2 \sqrt{d_{2}(\beta-\gamma-\delta)}$ and $d_{3}<2 d_{2}$, then (1.3) has a nontrivial nonnegative traveling wave solution satisfying $S(-\infty):=S_{-\infty}>S(+\infty):=S_{\infty} \geq 0, I( \pm \infty)=0$, $R(-\infty)=0$ and $R(+\infty)=\gamma\left(S_{-\infty}-S_{\infty}\right) /(\gamma+\delta)$; if $0<R_{0} \leq 1$ or $c<c^{*}$, then (1.3) admits no nontrivial nonnegative traveling waves. In reality, latent period of many diseases seems to be inevitable. Removing the unnatural condition $d_{3}<2 d_{2}$, He and Tsai 12 obtained the existence of nontrivial nonnegative traveling wave solutions with both super-critical and critical speeds for a discrete delayed version of $(1.3)$. For the investigation of other local-diffusion epidemic systems, we suggest the readers to see 1 , 
$68,10,13,14,22,23,25,28,32,37,40,43$. . To describe a long range process in a spatially continuous environment, Yang et al. [34 explored the nonlocal version of system (1.3)

$$
\left\{\begin{array}{l}
\partial_{t} S=d_{1}(J * S-S)-\beta S I /(S+I+R), \\
\partial_{t} I=d_{2}(J * I-I)+\beta S I /(S+I+R)-(\gamma+\delta) I, \\
\partial_{t} R=d_{3}(J * R-R)+\gamma I,
\end{array}\right.
$$

where " $*$ " is the standard convolution with respect to spatial variable, $J \in C^{1}(\mathbb{R}), J(x)=$ $J(-x) \geq 0, \int_{\mathbb{R}} J(y) d y=1$ and $J$ is compactly supported. They showed that when $R_{0}=\beta /(\gamma+\delta)>1$, there is a constant $c^{*}>0$ such that for every $c>c^{*}$, system (1.4) admits a nontrivial nonnegative traveling wave solution with $S(-\infty):=S_{-\infty}>S(+\infty):=$ $S_{\infty} \geq 0, I( \pm \infty)=0$ and $R(-\infty)=0$. Moreover, $R(+\infty)=\gamma\left(S_{-\infty}-S_{\infty}\right) /(\gamma+\delta)$ if $\lim \sup _{\xi \rightarrow+\infty} R(\xi)<+\infty$. When $0<R_{0} \leq 1$ or $0<c<c^{*}$, system (1.4) has no nontrivial nonnegative traveling waves. Very recently, Wei et al. 25] investigated a nonlocal delayed version of model (1.4) and derived the existence of nontrivial positive traveling waves with super-critical and critical speeds. For more study of nonlocal diffusion epidemic systems, we refer to $2,3,9,15,19,21,33,35,41,44]$. To study the nonlocal process in a spatially discrete environment, the present authors [26] proposed a two-component discrete diffusion epidemic model with delay

$$
\left\{\begin{array}{l}
\frac{d S_{n}(t)}{d t}=d_{1}\left[S_{n+1}(t)+S_{n-1}(t)-2 S_{n}(t)\right]-\frac{\beta S_{n}(t) I_{n}(t-\tau)}{S_{n}(t)+I_{n}(t-\tau)}, \\
\frac{d I_{n}(t)}{d t}=d_{2}\left[I_{n+1}(t)+I_{n-1}(t)-2 I_{n}(t)\right]+\frac{\beta S_{n}(t) I_{n}(t-\tau)}{S_{n}(t)+I_{n}(t-\tau)}-\gamma I_{n}(t), \quad n \in \mathbb{Z}
\end{array}\right.
$$

and established the existence and nonexistence of traveling wave solutions for this system. Let us recall the proof strategy in [26]. Firstly, we constructed a pair of super/sub-solutions on the real line and defined an invariant cone of a functional space with weighted norm by this pair of super-/sub-solutions. Secondly, we applied Schauder's fixed point theorem to prove that 1.5 has a traveling wave solution with super-critical speed. Thirdly, by analysis method we obtained the asymptotic boundary, positiveness and other properties of the traveling wave solution. Fourthly, utilizing the similar way as for the super-critical traveling wave solution, we still derived the critical traveling wave solution via another pair of super-/sub-solutions. Finally, for the nonexistence theorems, we mainly used the way of contradictory arguments and the bilateral Laplace transform to achieve the goal. The results in 26] are summarised as follows. If $\beta>\gamma$, then there is some constant $c^{*}>0$ such that for each $c \geq c^{*}$, model 1.5 admits a nontrivial positive bounded traveling wave solution. If $\beta \leq \gamma$ or $c<c^{*}$, then 1.5 has no nontrivial positive bounded traveling wave solutions. For other progress of discrete diffusion epidemic models, see [5, 11, 24, 30, 36, 42. 
We should point out that the difference-differential epidemic models in the existing references $[5,11,24,26,30,36,42]$ are two-component systems, while (1.1) is indeed a threecomponent system and we need to overcome some difficulties. Due to the deficiency of monotonicity for (1.1), it is hard to obtain the exact boundaries of $S$-component and $R$-component at plus infinity. However, by analysis technique, we still derive the existence of the limits for $S$-component and $R$-component at plus infinity under the condition $\lim \sup _{\xi \rightarrow+\infty} R(\xi)<+\infty$. Because of the appearance of second order difference operators, it seems difficult to deduce a priori estimate of $R$-component, which is a key estimate for using the method of the bilateral Laplace transform to prove the nonexistence results. Herein, we construct a nonnegative bounded smooth cut-off function and make full use the structure of system 1.2 to obtain this a priori estimate.

Now we sketch our ideas and organization as follows. Section 2 is devoted to stating some preliminaries. In Section 3, we apply the reduction to absurdity together with the bilateral Laplace transform to establish the nonexistence of nontrivial positive bounded traveling wave solutions in (1.1). In Section 4 to explore the existence of a super-critical traveling wave solution in (1.1), we first construct a pair of super-/subsolutions for 1.2); secondly, we introduce a convex cone $\Omega_{X}$ of initial functions defined in a large bounded closed interval $[-X, X]$, whose elements sandwich between supersolution and sub-solution; thirdly, we define a nonlinear operator $\mathcal{O}$ on $\Omega_{X}$ and present that $\mathcal{O}: \Omega_{X} \mapsto \Omega_{X}$ is completely continuous with respect to the supremum norm in $C\left([-X, X], \mathbb{R}^{3}\right)$; fourthly, we use Schauder's fixed point theorem on this cone to obtain the existence of a fixed point for $\mathcal{O}$, which guarantees that the existence of a solution for (1.2) on $[-X, X]$; fifthly, by a limiting method we extend the existence of the solution on $[-X, X]$ to the unbounded spatial domain $\mathbb{R}$; finally, by delicate analysis we show the positiveness and asymptotic boundaries of the traveling wave solutions. In Section 5 , to investigate the existence of a critical traveling wave solution in (1.1), we construct another pair of super-/sub-solutions for 1.2 and utilize the analogous manner as for the supercritical traveling wave solution to reach our goal. Then we further deduce some properties concerning the traveling wave solutions.

\section{Preliminaries}

Let us start with the definition of the super-/sub-solutions for 1.2 . In the sequel,

$$
D[u](\xi):=u(\xi+1)+u(\xi-1)-2 u(\xi) .
$$

Definition 2.1. The nonnegative continuous function pairs $\left(S_{+}, I_{+}, R_{+}\right)(\xi)$ and $\left(S_{-}, I_{-}\right.$, 
$\left.R_{-}\right)(\xi)$ are named as a pair of super-/sub-solutions for 1.2 if they satisfy

$$
\begin{gathered}
d_{s} D\left[S_{+}\right](\xi)-c S_{+}^{\prime}(\xi)-\frac{\beta S_{+}(\xi) I_{-}(\xi-c \tau)}{S_{+}(\xi)+I_{-}(\xi-c \tau)+R_{+}(\xi)} \leq 0, \\
d_{i} D\left[I_{+}\right](\xi)-c I_{+}^{\prime}(\xi)+\frac{\beta S_{+}(\xi) I_{+}(\xi-c \tau)}{S_{+}(\xi)+I_{+}(\xi-c \tau)+R_{-}(\xi)}-(\gamma+\delta) I_{+}(\xi) \leq 0, \\
d_{r} D\left[R_{+}\right](\xi)-c R_{+}^{\prime}(\xi)+\gamma I_{+}(\xi) \leq 0, \\
d_{s} D\left[S_{-}\right](\xi)-c S_{-}^{\prime}(\xi)-\frac{\beta S_{-}(\xi) I_{+}(\xi-c \tau)}{S_{-}(\xi)+I_{+}(\xi-c \tau)+R_{-}(\xi)} \geq 0, \\
d_{i} D\left[I_{-}\right](\xi)-c I_{-}^{\prime}(\xi)+\frac{\beta S_{-}(\xi) I_{-}(\xi-c \tau)}{S_{-}(\xi)+I_{-}(\xi-c \tau)+R_{+}(\xi)}-(\gamma+\delta) I_{-}(\xi) \geq 0, \\
d_{r} D\left[R_{-}\right](\xi)-c R_{-}^{\prime}(\xi)+\gamma I_{-}(\xi) \geq 0,
\end{gathered}
$$

except for finitely many points on the whole real line.

Now we establish a couple of lemmas which will be utilized to prove our main results.

Lemma 2.2. Let $R_{0}:=\beta /(\gamma+\delta)>1$ and

$$
F(\rho, c):=d_{i}\left(e^{\rho}+e^{-\rho}-2\right)-c \rho+\beta e^{-\rho c \tau}-\gamma-\delta .
$$

Then there exists a pair of positive real numbers $\left(\rho^{*}, c^{*}\right)$ such that

$$
F\left(\rho^{*}, c^{*}\right)=d_{i}\left(e^{\rho^{*}}+e^{-\rho^{*}}-2\right)-c^{*} \rho^{*}+\beta e^{-\rho^{*} c^{*} \tau}-\gamma-\delta=0
$$

and

$$
F_{\rho}\left(\rho^{*}, c^{*}\right)=d_{i}\left(e^{\rho^{*}}-e^{-\rho^{*}}\right)-c^{*}-\beta c^{*} \tau e^{-\rho^{*} c^{*} \tau}=0 .
$$

Moreover, the following statements are valid.

(i) If $c \in\left(0, c^{*}\right)$, then $F(\rho, c)>0$ for $\rho \in[0,+\infty)$.

(ii) If $c \in\left(c^{*},+\infty\right)$, then the equation $F(\rho, c)=0$ admits two positive roots $\rho_{1}(c):=\rho_{1}$ and $\rho_{2}(c):=\rho_{2}$ with $\rho^{*} \in\left(\rho_{1}, \rho_{2}\right)$ such that $F(\rho, c)>0$ for $\rho \in[0,+\infty) \backslash\left[\rho_{1}, \rho_{2}\right]$ and $F(\rho, c)<0$ for $\rho \in\left(\rho_{1}, \rho_{2}\right)$.

Proof. It follows that $F(+\infty, c)=+\infty$ for each $c>0$ and $F(\rho,+\infty)=-\infty$ for each $\rho>0$. Since $R_{0}>1$, we compute that

$$
\begin{gathered}
F(0, c)=\beta-\gamma-\delta>0, \quad F_{c}(\rho, c)=-\rho-\beta \rho \tau e^{-\rho c \tau}<0, \quad \forall \rho>0, \\
F(\rho, 0)=d_{i}\left(e^{\rho}+e^{-\rho}-2\right)+\beta-\gamma-\delta \geq \beta-\gamma-\delta>0, \\
F_{\rho}(0, c)=\left.\left(d_{i} e^{\rho}-d_{i} e^{-\rho}-c-\beta c \tau e^{-\rho c \tau}\right)\right|_{\rho=0}=-c-\beta c \tau<0, \quad \forall c>0
\end{gathered}
$$


and

$$
F_{\rho \rho}(\rho, c)=d_{i} e^{\rho}+d_{i} e^{-\rho}+\beta c^{2} \tau^{2} e^{-\rho c \tau}>0
$$

By these calculations, we show the rough graphs of function $F(\rho, c)$ for each $c>0$ in Figure 2.1.
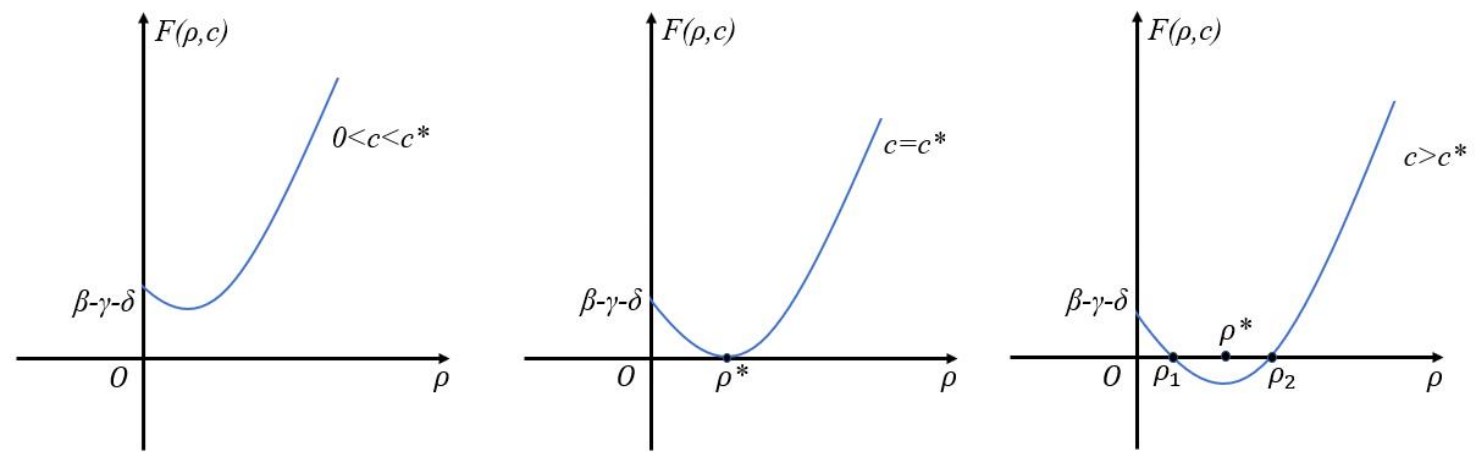

Figure 2.1: $F(\rho, c)$ when $0<c<c^{*}, c=c^{*}$ and $c>c^{*}$, respectively.

In view of this figure, we obtain the desired results of this lemma.

Lemma 2.3. Let

$$
G(\rho, c):=c \rho-d_{r}\left(e^{\rho}+e^{-\rho}-2\right)
$$

Then for each $c>0$, there exists a constant $\rho_{3}>0$ such that $G(\rho, c)>0$ with $\rho \in\left(0, \rho_{3}\right)$.

Proof. Elementary computations give that $G(0, c)=0, G_{\rho}(0, c)=c>0, G_{\rho \rho}(\rho, c)=$ $-d_{r}\left(e^{\rho}+e^{-\rho}\right)<0$ and $G(+\infty, c)=-\infty$ for each $c>0$. With the aid of the above computations, we present the rough graph of the function $G(\rho, c)$ for each $c>0$ in Figure 2.2 .

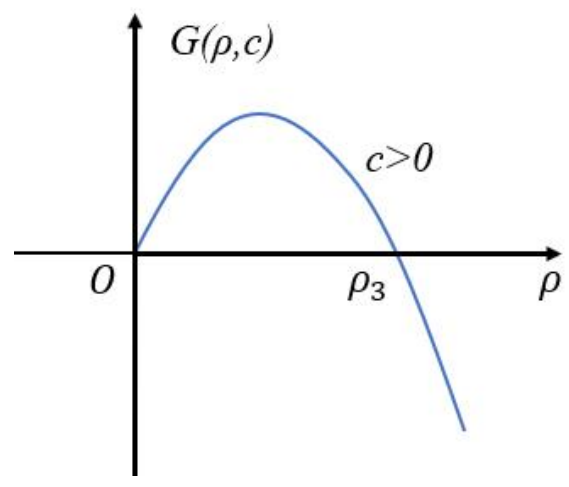

Figure 2.2: $G(\rho, c)$ when $c>0$.

By the virtue of this figure, we end the proof. 
Lemma 2.4. Suppose that $(S, I, R)(\xi) \in C^{1}\left(\mathbb{R}, \mathbb{R}^{3}\right)$ is a nontrivial positive solution of (1.2) with the wave speed $c>0$ and satisfies

$$
S(-\infty)=S_{1}, \quad \sup _{\xi \in \mathbb{R}} S(\xi) \leq S_{1}, \quad I( \pm \infty)=0, \quad R(-\infty)=0, \quad \sup _{\xi \in \mathbb{R}} R(\xi)<+\infty,
$$

where $S_{1}>0$ is a given constant. Then

$$
\int_{\mathbb{R}} \frac{S(\xi) I(\xi-c \tau)}{S(\xi)+I(\xi-c \tau)+R(\xi)} d \xi<+\infty
$$

and

$$
\int_{\mathbb{R}} I(\xi) d \xi<+\infty
$$

Proof. Integrating the first equation in 1.2 over $[x, y]$ gives

$$
\begin{aligned}
& \int_{x}^{y} \frac{\beta S(\xi) I(\xi-c \tau)}{S(\xi)+I(\xi-c \tau)+R(\xi)} d \xi \\
= & d_{s} \int_{x}^{y} D[S](\xi)-c \int_{x}^{y} S^{\prime}(\xi) d \xi \\
= & d_{s} \int_{x}^{y} \int_{0}^{1} S^{\prime}(\xi+\theta) d \theta d \xi-d_{s} \int_{x}^{y} \int_{0}^{1} S^{\prime}(\xi-\theta) d \theta d \xi-c S(y)+c S(x) \\
= & d_{s} \int_{0}^{1}[S(y+\theta)-S(x+\theta)] d \theta+d_{s} \int_{0}^{1}[S(x-\theta)-S(y-\theta)] d \theta-c S(y)+c S(x) \\
\leq & \left(2 d_{s}+c\right) S_{1}, \quad\left(\text { since } \sup _{\xi \in \mathbb{R}} S(\xi) \leq S_{1} \text { and } S(\xi)>0 \text { on } \mathbb{R}\right),
\end{aligned}
$$

which together with the positiveness of $S(\xi), I(\xi)$ and $R(\xi)$ on $\mathbb{R}$ implies that 2.3 holds. Since $I(\xi) \in C^{1}(\mathbb{R})$ is nontrivial, positive and $I( \pm \infty)=0$, there is some constant $C_{0}>0$ such that $I(\xi) \leq C_{0}$ on $\mathbb{R}$. Then an integration of the second equation in 1.2$)$ over $[\eta, \zeta]$ yields

$$
\begin{aligned}
& (\gamma+\delta) \int_{\eta}^{\zeta} I(\xi) d \xi \\
= & d_{i} \int_{\eta}^{\zeta} D[I](\xi)-c \int_{\eta}^{\zeta} I^{\prime}(\xi) d \xi+\int_{\eta}^{\zeta} \frac{\beta S(\xi) I(\xi-c \tau)}{S(\xi)+I(\xi-c \tau)+R(\xi)} d \xi \\
\leq & d_{i} \int_{\eta}^{\zeta} \int_{0}^{1} I^{\prime}(\xi+\theta) d \theta d \xi-d_{i} \int_{\eta}^{\zeta} \int_{0}^{1} I^{\prime}(\xi-\theta) d \theta d \xi-c I(\zeta)+c I(\eta)+\left(2 d_{s}+c\right) S_{1} \\
= & d_{i} \int_{0}^{1}[I(\zeta+\theta)-I(\eta+\theta)] d \theta+d_{i} \int_{0}^{1}[I(\eta-\theta)-I(\zeta-\theta)] d \theta \\
& -c I(\zeta)+c I(\eta)+\left(2 d_{s}+c\right) S_{1} \\
\leq & \left(2 d_{i}+c\right) C_{0}+\left(2 d_{s}+c\right) S_{1} .
\end{aligned}
$$

This combined with the positiveness of $I(\xi)$ on $\mathbb{R}$ ensures that 2.4 holds. The proof is finished. 


\section{Nonexistence of traveling waves}

This section is to establish the nonexistence of nontrivial positive bounded traveling wave solutions to (1.1).

Theorem 3.1. For a given constant $S_{1}>0$, if $\left(R_{0}, c\right) \in(0,1] \times \mathbb{R} \cup(1,+\infty) \times\left(-\infty, c^{*}\right)$, then system (1.1) has no nontrivial positive traveling wave solutions $(S, I, R)(\xi)$ satisfying

$$
S(-\infty)=S_{1}, \quad \sup _{\xi \in \mathbb{R}} S(\xi) \leq S_{1}, \quad I( \pm \infty)=0, \quad R(-\infty)=0, \quad \sup _{\xi \in \mathbb{R}} R(\xi)<+\infty .
$$

Proof. By the reduction to absurdity, we assume that $(S, I, R)(\xi) \in C^{1}\left(\mathbb{R}, \mathbb{R}^{3}\right)$ is a nontrivial positive traveling wave solution of (1.1) satisfying (3.1). Then we divide the proof into three cases.

First case: $R_{0} \leq 1$ and $c \in \mathbb{R}$. An integration of the second equation in $(1.2)$ over $\mathbb{R}$ yields that

$$
\begin{aligned}
(\gamma+\delta) \int_{\mathbb{R}} I(\xi) d \xi & =d_{i} \int_{\mathbb{R}} D[I](\xi) d \xi-c \int_{\mathbb{R}} I^{\prime}(\xi) d \xi+\int_{\mathbb{R}} \frac{\beta S(\xi) I(\xi-c \tau)}{S(\xi)+I(\xi-c \tau)+R(\xi)} d \xi \\
& \left.=\int_{\mathbb{R}} \frac{\beta S(\xi) I(\xi-c \tau)}{S(\xi)+I(\xi-c \tau)+R(\xi)} d \xi \quad(\text { by } \quad 2.4) \text { and } I( \pm \infty)=0\right) \\
& \left.<\beta \int_{\mathbb{R}} I(\xi-c \tau) d \xi \quad \text { (by the positiveness of } S, I, R \text { on } \mathbb{R}\right) \\
& \leq(\gamma+\delta) \int_{\mathbb{R}} I(\xi) d \xi \quad\left(\text { since } R_{0} \leq 1\right) .
\end{aligned}
$$

A contradiction appears.

Second case: $R_{0}>1$ and $0<c<c^{*}$. It follows from 3.1 that

$$
\frac{\beta S(\xi)}{S(\xi)+I(\xi-c \tau)+R(\xi)} \rightarrow \beta \quad \text { as } \xi \rightarrow-\infty .
$$

By (3.2) and $R_{0}>1$, we have that there is a constant $\xi^{*} \ll 0$ such that

$$
\frac{\beta S(\xi)}{S(\xi)+I(\xi-c \tau)+R(\xi)}>\frac{\beta+\gamma+\delta}{2} \text { for } \xi<\xi^{*} .
$$

Then from $(3.3)$ and the second equation in $(1.2)$, we deduce that

$$
c I^{\prime}(\xi) \geq d_{i} D[I](\xi)+\frac{\beta+\gamma+\delta}{2}[I(\xi-c \tau)-I(\xi)]+\frac{\beta-\gamma-\delta}{2} I(\xi) \quad \text { for } \xi<\xi^{*} .
$$

Using (2.4) we define the improper integral

$$
H(\xi):=\int_{-\infty}^{\xi} I(\eta) d \eta \quad \text { for } \xi \in \mathbb{R} .
$$


Integrating (3.4) over $(-\infty, \xi]$ gives

$$
\frac{\beta-\gamma-\delta}{2} H(\xi) \leq c I(\xi)-d_{i} D[H](\xi)-\frac{\beta+\gamma+\delta}{2}[H(\xi-c \tau)-H(\xi)] \text { for } \xi<\xi^{*},
$$

where we have used $I(-\infty)=0$. By dominated convergence theorem and $H(-\infty)=0$, we obtain

$$
\begin{aligned}
& \int_{-\infty}^{\xi} D[H](\xi) d \eta \\
= & \lim _{z \rightarrow-\infty} \int_{z}^{\xi}[H(\eta+1)-H(\eta)] d \eta+\lim _{z \rightarrow-\infty} \int_{z}^{\xi}[H(\eta-1)-H(\eta)] d \eta \\
= & \lim _{z \rightarrow-\infty} \int_{z}^{\xi} \int_{0}^{1} H^{\prime}(\eta+\theta) d \theta d \eta-\lim _{z \rightarrow-\infty} \int_{z}^{\xi} \int_{0}^{1} H^{\prime}(\eta-\theta) d \theta d \eta \\
= & \lim _{z \rightarrow-\infty} \int_{0}^{1}[H(\xi+\theta)-H(z+\theta)] d \theta-\lim _{z \rightarrow-\infty} \int_{0}^{1}[H(\xi-\theta)-H(z-\theta)] d \theta \\
= & \int_{0}^{1}[H(\xi+\theta)-H(\xi-\theta)] d \theta
\end{aligned}
$$

and

$$
\begin{aligned}
\int_{-\infty}^{\xi}[H(\eta-c \tau)-H(\eta)] d \eta & =\lim _{z \rightarrow-\infty} \int_{z}^{\xi}[H(\eta-c \tau)-H(\eta)] d \eta \\
& =-c \tau \lim _{z \rightarrow-\infty} \int_{z}^{\xi} \int_{0}^{1} H^{\prime}(\eta-c \tau \theta) d \theta d \eta \\
& =-c \tau \lim _{z \rightarrow-\infty} \int_{0}^{1}[H(\xi-c \tau \theta)-H(z-c \tau \theta)] d \theta \\
& =-c \tau \int_{0}^{1} H(\xi-c \tau \theta) d \theta
\end{aligned}
$$

which guarantee that $H(\xi+1)+H(\xi-1)-2 H(\xi)$ and $H(\xi-c \tau)-H(\xi)$ are integrable on $\mathbb{R}$. Thus integrating (3.5) on $(-\infty, \xi]$ and utilizing (3.6) and (3.7) yield that

$$
\begin{aligned}
& \frac{\beta-\gamma-\delta}{2} \int_{-\infty}^{\xi} H(\eta) d \eta+d_{i} \int_{0}^{1}[H(\xi+\theta)-H(\xi-\theta)] d \theta \\
\leq & c H(\xi)+\frac{c \tau(\beta+\gamma+\delta)}{2} \int_{0}^{1} H(\xi-c \tau \theta) d \theta \quad \text { for } \xi<\xi^{*} .
\end{aligned}
$$

Note that $H(\xi)$ is strictly increasing on $\mathbb{R}$ due to the positiveness of $I(\xi)$ on $\mathbb{R}$. Then it follows from (3.8) that

$$
\frac{\beta-\gamma-\delta}{2} \int_{-\infty}^{\xi} H(\eta) d \eta \leq\left[c+\frac{c \tau(\beta+\gamma+\delta)}{2}\right] H(\xi) \text { for } \xi<\xi^{*} .
$$

From (3.9) and the monotonicity of $H(\xi)$, we obtain that there is a large enough constant $\eta_{0}>0$ such that

$$
\frac{\eta_{0}(\beta-\gamma-\delta)}{2} H\left(\xi-\eta_{0}\right) \leq\left[c+\frac{c \tau(\beta+\gamma+\delta)}{2}\right] H(\xi) \text { for } \xi<\xi^{*},
$$


which implies that

$$
H\left(\xi-\eta_{0}\right) \leq \sigma H(\xi) \text { for } \xi<\xi^{*}
$$

where $\sigma \in(0,1)$ is a small enough constant. Denote

$$
\mu_{0}:=\frac{1}{\eta_{0}} \ln \frac{1}{\sigma} \quad \text { and } \quad J(\xi):=H(\xi) e^{-\mu_{0} \xi}
$$

We infer from (3.10) and 3.11 that

$$
J\left(\xi-\eta_{0}\right)=H\left(\xi-\eta_{0}\right) e^{-\mu_{0}\left(\xi-\eta_{0}\right)} \leq \sigma H(\xi) e^{-\mu_{0}\left(\xi-\eta_{0}\right)}=J(\xi) \text { for } \xi<\xi^{*},
$$

which coupled with $J(\xi)>0$ ensures that $J(-\infty)$ exists. Also it is easy to see that $J(+\infty)=0$. Hence there exists a constant $J_{0}>0$ such that

$$
J(\xi) \leq J_{0} \quad \text { for } \xi \in \mathbb{R}
$$

Obviously, one can have from the second equation in 1.2 that

$$
c I^{\prime}(\xi) \leq d_{i} D[I](\xi)+\beta I(\xi-c \tau)-(\gamma+\delta) I(\xi)
$$

Integrating (3.13) over $(-\infty, \xi]$ leads to

$$
c I(\xi) \leq d_{i} D[H](\xi)+\beta H(\xi-c \tau)-(\gamma+\delta) H(\xi)
$$

Then it follows from $3.12-3.14$ that

$$
\sup _{\xi \in \mathbb{R}}\left\{I(\xi) e^{-\mu_{0} \xi}\right\}<+\infty \text { and } \sup _{\xi \in \mathbb{R}}\left\{I^{\prime}(\xi) e^{-\mu_{0} \xi}\right\}<+\infty \text {. }
$$

Let $v(\xi) \in C^{\infty}(\mathbb{R},[0,1])$ be a nondecreasing function such that $v_{N}(\xi)=v(\xi / N)$ for $N \in \mathbb{N}$ and

$$
v(\xi)= \begin{cases}0 & \text { if } \xi \in(-\infty,-2] \\ 1 & \text { if } \xi \in[-1,+\infty)\end{cases}
$$

Multiplying the third equation in $(1.2)$ by $e^{-\nu \xi} v_{N}(\xi)$ and integrating the resultant equation over $\mathbb{R}$, we obtain

$$
c \int_{\mathbb{R}} R^{\prime}(\xi) e^{-\nu \xi} v_{N}(\xi) d \xi=d_{r} \int_{\mathbb{R}} D[R](\xi) e^{-\nu \xi} v_{N}(\xi) d \xi+\gamma \int_{\mathbb{R}} I(\xi) e^{-\nu \xi} v_{N}(\xi) d \xi
$$

By direct calculations, we deduce that

$$
\int_{\mathbb{R}} R^{\prime}(\xi) e^{-\nu \xi} v_{N}(\xi) d \xi=\nu \int_{\mathbb{R}} R(\xi) e^{-\nu \xi} v_{N}(\xi) d \xi-\int_{\mathbb{R}} R(\xi) e^{-\nu \xi} v_{N}^{\prime}(\xi) d \xi
$$


and

$$
\begin{aligned}
& \int_{\mathbb{R}}[R(\xi+1)+R(\xi-1)] e^{-\nu \xi} v_{N}(\xi) d \xi \\
= & \int_{\mathbb{R}} R(\xi+1) e^{-\nu \xi} v_{N}(\xi) d \xi+\int_{\mathbb{R}} R(\xi-1) e^{-\nu \xi} v_{N}(\xi) d \xi \\
= & e^{\nu} \int_{\mathbb{R}} R(\xi) e^{-\nu \xi} v_{N}(\xi-1) d \xi+e^{-\nu} \int_{\mathbb{R}} R(\xi) e^{-\nu \xi} v_{N}(\xi+1) d \xi \\
\leq & \left(e^{\nu}+e^{-\nu}\right) \int_{\mathbb{R}} R(\xi) e^{-\nu \xi} d \xi
\end{aligned}
$$

since $v_{N}(\xi-1) \leq 1$ and $v_{N}(\xi+1) \leq 1$ for $\xi \in \mathbb{R}$. Plugging 3.17) and 3.18 into 3.16 yields

$$
\begin{aligned}
& \left(c \nu+2 d_{r}\right) \int_{\mathbb{R}} R(\xi) e^{-\nu \xi} v_{N}(\xi) d \xi-d_{r}\left(e^{\nu}+e^{-\nu}\right) \int_{\mathbb{R}} R(\xi) e^{-\nu \xi} d \xi-c \int_{\mathbb{R}} R(\xi) e^{-\nu \xi} v_{N}^{\prime}(\xi) d \xi \\
\leq & \gamma \int_{\mathbb{R}} I(\xi) e^{-\nu \xi} v_{N}(\xi) d \xi .
\end{aligned}
$$

Recall that $G(\nu, c)=c \nu+2 d_{r}-d_{r}\left(e^{\nu}+e^{-\nu}\right)>0$ for each $\nu \in\left(0, \rho_{3}\right)$ (see Lemma 2.3). Then passing to the limits in $(3.19)$ as $N \rightarrow \infty$ gives

$$
\int_{\mathbb{R}} R(\xi) e^{-\nu \xi} d \xi \leq \frac{\gamma}{G(\nu, c)} \int_{\mathbb{R}} I(\xi) e^{-\nu \xi} d \xi \quad \text { for } \nu \in\left(0, \rho_{3}\right) .
$$

Hence by 3.15 we obtain that

$$
\int_{\mathbb{R}} R(\xi) e^{-\nu \xi} d \xi<+\infty \quad \text { for } \nu \in(0, \underline{\mu}) \text { with } \underline{\mu}:=\min \left\{\mu_{0}, \rho_{3}\right\} .
$$

It follows from 3.15 and 3.20 that

$$
\int_{\mathbb{R}} e^{-\rho \xi} \frac{I(\xi-c \tau)[I(\xi-c \tau)+R(\xi)]}{S(\xi)+I(\xi-c \tau)+R(\xi)} d \xi<+\infty \quad \text { for } \rho \in\left(0, \mu_{0}+\underline{\mu}\right) .
$$

By (3.15) and the boundedness of $I(\xi)$ on $\mathbb{R}$, we define the two-sided Laplace transform of $I(\xi)$ by

$$
L(\rho):=\int_{\mathbb{R}} I(\xi) e^{-\rho \xi} d \xi \quad \text { with } 0<\operatorname{Re} \rho<\mu_{0} .
$$

Note that the second equation in 1.2 can be rewritten as

$$
d_{i} D[I](\xi)-c I^{\prime}(\xi)+\beta I(\xi-c \tau)-(\gamma+\delta) I(\xi)=\frac{\beta I(\xi-c \tau)[I(\xi-c \tau)+R(\xi)]}{S(\xi)+I(\xi-c \tau)+R(\xi)}
$$

By taking the two-sided Laplace transform on (3.22), we have

$$
L(\rho) F(\rho, c)=\int_{\mathbb{R}} e^{-\rho \xi} \frac{\beta I(\xi-c \tau)[I(\xi-c \tau)+R(\xi)]}{S(\xi)+I(\xi-c \tau)+R(\xi)} d \xi .
$$


One can see that $L(\rho)$ on the left-hand side in 3.23$)$ is well-defined for $\rho \in\left(0, \mu_{0}\right)$, while

$$
\int_{\mathbb{R}} e^{-\rho \xi} \frac{\beta I(\xi-c \tau)[I(\xi-c \tau)+R(\xi)]}{S(\xi)+I(\xi-c \tau)+R(\xi)} d \xi
$$

on the right-hand side is well-defined for $\rho \in\left(0, \mu_{0}+\mu\right)$ (see (3.21)). According to the property of Laplace transform [29], we obtain that these two integrals are analytical on the whole right half plane; see the analogously discussions in $12,20,22,23,26,27,40,41,44]$. Due to $F(\rho, c) \rightarrow+\infty$ as $\rho \rightarrow+\infty$ (see the proof in Lemma 2.2), a contradiction occurs in 3.23 .

Third case: $R_{0}>1$ and $c \leq 0$. From $(3.8)$, we get

$$
\begin{aligned}
& \frac{\beta-\gamma-\delta}{2} \int_{-\infty}^{\xi} H(\eta) d \eta+d_{i} \int_{0}^{1}[H(\xi+\theta)-H(\xi-\theta)] d \theta \\
\leq & c H(\xi)+\frac{c \tau(\beta+\gamma+\delta)}{2} \int_{0}^{1} H(\xi-c \tau \theta) d \theta \quad \text { for } \xi<\xi^{*} .
\end{aligned}
$$

With aid of the positiveness and monotonicity of $H(\xi)$ on $\mathbb{R}$, we conclude that inequality (3.24) does not hold under the conditions $R_{0}>1$ and $c \leq 0$, since the left-hand side in (3.24) is greater than zero, while the right-hand side in (3.24) is less than or equal to zero. A contradiction appears. Combining the above three cases, we finish the proof.

\section{Existence of super-critical traveling waves}

In this section, we shall prove the existence result under the conditions $R_{0}>1$ and $c>c^{*}$. For this purpose, recalling the definition of $\rho_{1}$ in Lemma 2.2, we select a constant $\rho_{4} \in\left(0, \rho_{1}\right)$ to be small enough such that

$$
M_{1}:=S_{1}+\frac{\beta e^{-\rho_{1} c \tau}}{d_{s}\left(2-e^{\rho_{4}}-e^{-\rho_{4}}\right)+c \rho_{4}}>S_{1},
$$

where $S_{1}>0$ is a given constant. Then choose a constant $\sigma_{1}>0$ such that the following algebraic equation

$$
S_{1}-M_{1} e^{\rho_{4} \xi}=\sigma_{1} e^{-\frac{\beta}{c} \xi}
$$

admits two negative roots and we denote the bigger one by $\xi_{1}$. Set

$$
\xi_{2}:=\frac{1}{\rho_{1}} \ln \left[\left(R_{0}-1\right) S_{1}\right] \quad \text { and } \quad \xi_{3}:=\frac{1}{\epsilon_{1}} \ln \frac{1}{M_{2}},
$$

where the constants $\epsilon_{1} \in\left(0, \min \left\{\rho_{2}-\rho_{1}, \rho_{3}, \rho_{4}\right\}\right)$ is chosen to be sufficiently small and $M_{2} \gg 1$ such that

$$
\xi_{3}<\xi_{2}, \quad \xi_{3}<\xi_{1} \quad \text { and } \quad S_{1}-M_{1} e^{\rho_{4} \xi_{3}} \geq S_{1} / 2
$$


Also we pick the constant

$$
M_{3} \geq \max \left\{\frac{\gamma e^{\left(\rho_{1}-\epsilon_{1}\right) \xi_{2}}}{G\left(\epsilon_{1}, c\right)}, \frac{\gamma\left(R_{0}-1\right) S_{1} e^{-\epsilon_{1} \xi_{2}}}{G\left(\epsilon_{1}, c\right)}\right\}
$$

where $G(\cdot, \cdot)$ is defined in Lemma 2.3. By the choices of above parameters, we construct the following nonnegative continuous functions on $\mathbb{R}$.

$$
\begin{aligned}
S_{+}(\xi):=S_{1}, & S_{-}(\xi):= \begin{cases}S_{1}-M_{1} e^{\rho_{4} \xi}, & \xi<\xi_{1}, \\
\sigma_{1} e^{-\frac{\beta}{c} \xi}, & \xi \geq \xi_{1},\end{cases} \\
I_{+}(\xi):=\left\{\begin{array}{lll}
e^{\rho_{1} \xi}, & \xi<\xi_{2}, \\
\left(R_{0}-1\right) S_{1}, & \xi \geq \xi_{2},
\end{array}\right. & I_{-}(\xi):= \begin{cases}e^{\rho_{1} \xi}-M_{2} e^{\left(\rho_{1}+\epsilon_{1}\right) \xi}, & \xi<\xi_{3}, \\
0, & \xi \geq \xi_{3},\end{cases} \\
R_{+}(\xi):=M_{3} e^{\epsilon_{1} \xi}, & R_{-}(\xi):=0 .
\end{aligned}
$$

In order to describe that the parameters are admissible, we show Figures $4.1,4.2$ and 4.3 .
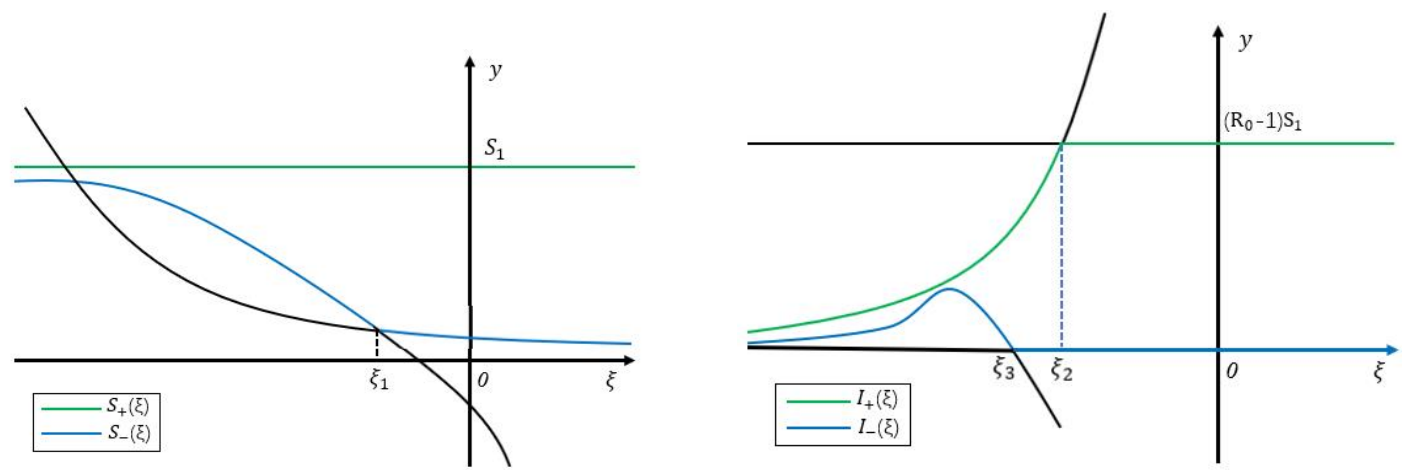

Figure 4.1: $S_{+}(\xi)$ and $S_{-}(\xi)$ when $R_{0}>1$ Figure 4.2: $I_{+}(\xi)$ and $I_{-}(\xi)$ when $R_{0}>1$ and $c>c^{*}$. and $c>c^{*}$.

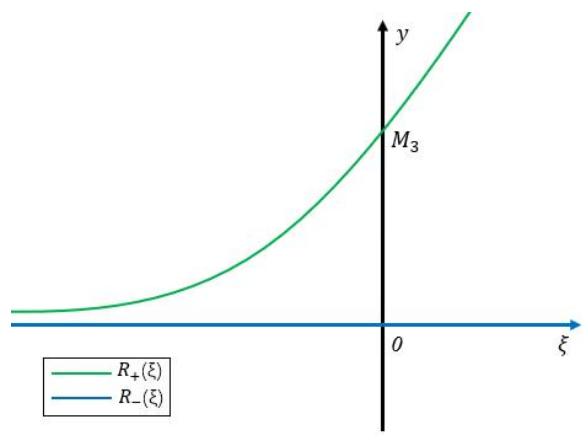

Figure 4.3: $R_{+}(\xi)$ and $R_{-}(\xi)$ when $R_{0}>1$ and $c>c^{*}$. 
Lemma 4.1. The functions $S_{ \pm}(\xi), I_{ \pm}(\xi)$ and $R_{ \pm}(\xi)$ satisfy

$$
\begin{gathered}
d_{s} D\left[S_{+}\right](\xi)-c S_{+}^{\prime}(\xi)-\frac{\beta S_{+}(\xi) I_{-}(\xi-c \tau)}{S_{+}(\xi)+I_{-}(\xi-c \tau)+R_{+}(\xi)} \leq 0, \quad \xi \in \mathbb{R}, \\
d_{i} D\left[I_{+}\right](\xi)-c I_{+}^{\prime}(\xi)+\frac{\beta S_{+}(\xi) I_{+}(\xi-c \tau)}{S_{+}(\xi)+I_{+}(\xi-c \tau)+R_{-}(\xi)}-(\gamma+\delta) I_{+}(\xi) \leq 0, \quad \xi \neq \xi_{2}, \\
d_{r} D\left[R_{+}\right](\xi)-c R_{+}^{\prime}(\xi)+\gamma I_{+}(\xi) \leq 0, \quad \xi \in \mathbb{R}, \\
d_{s} D\left[S_{-}\right](\xi)-c S_{-}^{\prime}(\xi)-\frac{\beta S_{-}(\xi) I_{+}(\xi-c \tau)}{S_{-}(\xi)+I_{+}(\xi-c \tau)+R_{-}(\xi)} \geq 0, \quad \xi \neq \xi_{1}, \\
d_{i} D\left[I_{-}\right](\xi)-c I_{-}^{\prime}(\xi)+\frac{\beta S_{-}(\xi) I_{-}(\xi-c \tau)}{S_{-}(\xi)+I_{-}(\xi-c \tau)+R_{+}(\xi)}-(\gamma+\delta) I_{-}(\xi) \geq 0, \quad \xi \neq \xi_{3}, \\
d_{r} D\left[R_{-}\right](\xi)-c R_{-}^{\prime}(\xi)+\gamma I_{-}(\xi) \geq 0, \quad \xi \in \mathbb{R} .
\end{gathered}
$$

Proof. From the definitions of $S_{+}(\xi), I_{-}(\xi)$ and $R_{ \pm}(\xi)$ on $\mathbb{R}$, we obtain that inequalities (4.4) and (4.9) hold naturally. Subsequently, we give the detailed proofs of inequalities 4.5-4.8.

Proof of 4.5 . If $\xi<\xi_{2}$, then $I_{+}(\xi)=e^{\rho_{1} \xi}, I_{+}(\xi-1)=e^{\rho_{1}(\xi-1)}, I_{+}(\xi-c \tau)=e^{\rho_{1}(\xi-c \tau)}$ and $I_{+}(\xi+1) \leq e^{\rho_{1}(\xi+1)}$. It follows from Lemma 2.2 that

$$
\begin{aligned}
& d_{i} D\left[I_{+}\right](\xi)-c I_{+}^{\prime}(\xi)+\frac{\beta S_{+}(\xi) I_{+}(\xi-c \tau)}{S_{+}(\xi)+I_{+}(\xi-c \tau)+R_{-}(\xi)}-(\gamma+\delta) I_{+}(\xi) \\
\leq & d_{i} D\left[I_{+}\right](\xi)-c I_{+}^{\prime}(\xi)+\beta I_{+}(\xi-c \tau)-(\gamma+\delta) I_{+}(\xi) \\
\leq & e^{\rho_{1} \xi}\left[d_{i}\left(e^{\rho_{1}}+e^{-\rho_{1}}-2\right)-c \rho_{1}+\beta e^{-\rho_{1} c \tau}-\gamma-\delta\right] \\
= & e^{\rho_{1} \xi} F\left(\rho_{1}, c\right)=0 .
\end{aligned}
$$

If $\xi>\xi_{2}$, then $I_{+}(\xi)=I_{+}(\xi+1)=\left(R_{0}-1\right) S_{1}, I_{+}(\xi-1) \leq\left(R_{0}-1\right) S_{1}, I_{+}(\xi-c \tau) \leq$ $\left(R_{0}-1\right) S_{1}, S_{+}(\xi)=S_{1}$ and $R_{-}(\xi)=0$. A direct computation leads to

$$
\begin{aligned}
& d_{i} D\left[I_{+}\right](\xi)-c I_{+}^{\prime}(\xi)+\frac{\beta S_{+}(\xi) I_{+}(\xi-c \tau)}{S_{+}(\xi)+I_{+}(\xi-c \tau)+R_{-}(\xi)}-(\gamma+\delta) I_{+}(\xi) \\
\leq & \frac{\beta S_{1}\left(R_{0}-1\right) S_{1}}{S_{1}+\left(R_{0}-1\right) S_{1}}-(\gamma+\delta)\left(R_{0}-1\right) S_{1}=0 .
\end{aligned}
$$

Proof of 4.6 . By the definitions of $I_{+}(\xi)$ and $R_{+}(\xi)$ on $\mathbb{R}$, we derive from Lemma 2.3 and 4.3 that

$$
\begin{aligned}
& d_{r} D\left[R_{+}\right](\xi)-c R_{+}^{\prime}(\xi)+\gamma I_{+}(\xi) \\
= & d_{r}\left[M_{3} e^{\epsilon_{1}(\xi+1)}+M_{3} e^{\epsilon_{1}(\xi-1)}-2 M_{3} e^{\epsilon_{1} \xi}\right]-c M_{3} \epsilon_{1} e^{\epsilon_{1} \xi}+\gamma e^{\rho_{1} \xi} \\
= & M_{3} e^{\epsilon_{1} \xi}\left[d_{r}\left(e^{\epsilon_{1}}+e^{-\epsilon_{1}}-2\right)-c \epsilon_{1}+\frac{\gamma e^{\left(\rho_{1}-\epsilon_{1}\right) \xi}}{M_{3}}\right] \\
\leq & M_{3} e^{\epsilon_{1} \xi}\left[\frac{\gamma e^{\left(\rho_{1}-\epsilon_{1}\right) \xi_{2}}}{M_{3}}-G\left(\epsilon_{1}, c\right)\right] \leq 0 \quad \text { for } \xi<\xi_{2}
\end{aligned}
$$


and

$$
\begin{aligned}
& d_{r} D\left[R_{+}\right](\xi)-c R_{+}^{\prime}(\xi)+\gamma I_{+}(\xi) \\
= & d_{r}\left[M_{3} e^{\epsilon_{1}(\xi+1)}+M_{3} e^{\epsilon_{1}(\xi-1)}-2 M_{3} e^{\epsilon_{1} \xi}\right]-c M_{3} \epsilon_{1} e^{\epsilon_{1} \xi}+\gamma\left(R_{0}-1\right) S_{1} \\
= & M_{3} e^{\epsilon_{1} \xi}\left[d_{r}\left(e^{\epsilon_{1}}+e^{-\epsilon_{1}}-2\right)-c \epsilon_{1}+\frac{\gamma\left(R_{0}-1\right) S_{1} e^{-\epsilon_{1} \xi}}{M_{3}}\right] \\
\leq & M_{3} e^{\epsilon_{1} \xi}\left[\frac{\gamma\left(R_{0}-1\right) S_{1} e^{-\epsilon_{1} \xi_{2}}}{M_{3}}-G\left(\epsilon_{1}, c\right)\right] \leq 0 \quad \text { for } \xi \geq \xi_{2} .
\end{aligned}
$$

Proof of 4.7). If $\xi<\xi_{1}$, then $S_{-}(\xi)=S_{1}-M_{1} e^{\rho_{4} \xi}, S_{-}(\xi-1)=S_{1}-M_{1} e^{\rho_{4}(\xi-1)}$, $S_{-}(\xi+1) \geq S_{1}-M_{1} e^{\rho_{4}(\xi+1)}, I_{+}(\xi-c \tau) \leq e^{\rho_{1}(\xi-c \tau)}$ and $R_{-}(\xi)=0$. By 4.1 we compute that

$$
\begin{aligned}
& d_{s} D\left[S_{-}\right](\xi)-c S_{-}^{\prime}(\xi)-\frac{\beta S_{-}(\xi) I_{+}(\xi-c \tau)}{S_{-}(\xi)+I_{+}(\xi-c \tau)+R_{-}(\xi)} \\
\geq & d_{s} D\left[S_{-}\right](\xi)-c S_{-}^{\prime}(\xi)-\beta I_{+}(\xi-c \tau) \\
\geq & d_{s}\left[2 M_{1} e^{\rho_{4} \xi}-M_{1} e^{\rho_{4}(\xi+1)}-M_{1} e^{\rho_{4}(\xi-1)}\right]+c M_{1} \rho_{4} e^{\rho_{4} \xi}-\beta e^{\rho_{1}(\xi-c \tau)} \\
= & e^{\rho_{4} \xi}\left[M_{1} d_{s}\left(2-e^{\rho_{4}}-e^{-\rho_{4}}\right)+c M_{1} \rho_{4}-\beta e^{\left(\rho_{1}-\rho_{4}\right) \xi-\rho_{1} c \tau}\right] \\
\geq & e^{\rho_{4} \xi}\left\{M_{1}\left[d_{s}\left(2-e^{\rho_{4}}-e^{-\rho_{4}}\right)+c \rho_{4}\right]-\beta e^{-\rho_{1} c \tau}\right\} \\
\geq & 0 \quad \text { for } \xi<\xi_{1},
\end{aligned}
$$

where we have used the fact that $e^{\left(\rho_{1}-\rho_{4}\right) \xi}<1$ for $\xi<\xi_{1}<0$. If $\xi>\xi_{1}$, then $S_{-}(\xi)=$ $\sigma_{1} e^{-\frac{\beta}{c} \xi}, S_{-}(\xi+1)=\sigma_{1} e^{-\frac{\beta}{c}(\xi+1)}$ and $S_{-}(\xi-1) \geq \sigma_{1} e^{-\frac{\beta}{c}(\xi-1)}$. We obtain that

$$
\begin{aligned}
& d_{s} D\left[S_{-}\right](\xi)-c S_{-}^{\prime}(\xi)-\frac{\beta S_{-}(\xi) I_{+}(\xi-c \tau)}{S_{-}(\xi)+I_{+}(\xi-c \tau)+R_{-}(\xi)} \\
\geq & d_{s} D\left[S_{-}\right](\xi)-c S_{-}^{\prime}(\xi)-\beta S_{-}(\xi) \\
\geq & d_{s}\left[\sigma_{1} e^{-\frac{\beta}{c}(\xi+1)}+\sigma_{1} e^{-\frac{\beta}{c}(\xi-1)}-2 \sigma_{1} e^{-\frac{\beta}{c} \xi}\right]+\beta \sigma_{1} e^{-\frac{\beta}{c} \xi}-\beta \sigma_{1} e^{-\frac{\beta}{c} \xi} \\
= & d_{s} \sigma_{1} e^{-\frac{\beta}{c} \xi}\left(e^{-\beta / c}+e^{\beta / c}-2\right) \\
\geq & 0 \text { for } \xi>\xi_{1} .
\end{aligned}
$$

Proof of (4.8). By 4.2) we get for $\xi<\xi_{3}$ that

$$
I_{-}(\xi)=e^{\rho_{1} \xi}-M_{2} e^{\left(\rho_{1}+\epsilon_{1}\right) \xi}, \quad S_{-}(\xi)=S_{1}-M_{1} e^{\rho_{4} \xi} \geq S_{1} / 2 \quad \text { and } \quad R_{+}(\xi)=M_{3} e^{\epsilon_{1} \xi} .
$$

For $\xi<\xi_{3}$, we deduce from 4.10 that

$$
\begin{aligned}
& -\beta I_{-}(\xi-c \tau)+\frac{\beta S_{-}(\xi) I_{-}(\xi-c \tau)}{S_{-}(\xi)+I_{-}(\xi-c \tau)+R_{+}(\xi)} \\
= & -\frac{\beta I_{-}^{2}(\xi-c \tau)+\beta I_{-}(\xi-c \tau) R_{+}(\xi)}{S_{-}(\xi)+I_{-}(\xi-c \tau)+R_{+}(\xi)} \geq-\frac{\beta I_{-}^{2}(\xi-c \tau)+\beta I_{-}(\xi-c \tau) R_{+}(\xi)}{S_{-}(\xi)} \\
\geq & -\frac{2 \beta}{S_{1}}\left[e^{2 \rho_{1} \xi}+M_{3} e^{\left(\rho_{1}+\epsilon_{1}\right) \xi}\right]
\end{aligned}
$$


since $I_{-}(\xi-c \tau) \leq I_{+}(\xi)=e^{\rho_{1} \xi}$ for $\xi<\xi_{3}$. Recalling that $\epsilon_{1} \in\left(0, \min \left\{\rho_{2}-\rho_{1}, \rho_{3}, \rho_{4}\right\}\right)$, we have for $\xi<\xi_{3}<0$ that

$$
e^{\left(\rho_{1}-\epsilon_{1}\right) \xi}<1, \quad F\left(\rho_{1}, c\right)=0 \quad \text { and } \quad F\left(\rho_{1}+\epsilon_{1}, c\right)<0 .
$$

Noting that $M_{2} \gg 1$ and using (4.11) and 4.12, we derive for $\xi<\xi_{3}$ that

$$
\begin{aligned}
& d_{i} D\left[I_{-}\right](\xi)-c I_{-}^{\prime}(\xi)+\frac{\beta S_{-}(\xi) I_{-}(\xi-c \tau)}{S_{-}(\xi)+I_{-}(\xi-c \tau)+R_{+}(\xi)}-(\gamma+\delta) I_{-}(\xi) \\
= & d_{i} D\left[I_{-}\right](\xi)-c I_{-}^{\prime}(\xi)+\beta I_{-}(\xi-c \tau)-(\gamma+\delta) I_{-}(\xi)-\beta I_{-}(\xi-c \tau) \\
& +\frac{\beta S_{-}(\xi) I_{-}(\xi-c \tau)}{S_{-}(\xi)+I_{-}(\xi-c \tau)+R_{+}(\xi)} \\
\geq & e^{\rho_{1} \xi} F\left(\rho_{1}, c\right)-M_{2} e^{\left(\rho_{1}+\epsilon_{1}\right) \xi} F\left(\rho_{1}+\epsilon_{1}, c\right)-\frac{2 \beta}{S_{1}}\left[e^{2 \rho_{1} \xi}+M_{3} e^{\left(\rho_{1}+\epsilon_{1}\right) \xi}\right] \\
= & -e^{\left(\rho_{1}+\epsilon_{1}\right) \xi} F\left(\rho_{1}+\epsilon_{1}, c\right)\left[M_{2}-\frac{2 \beta e^{\left(\rho_{1}-\epsilon_{1}\right) \xi}+2 \beta M_{3}}{-S_{1} F\left(\rho_{1}+\epsilon_{1}, c\right)}\right] \\
\geq & -e^{\left(\rho_{1}+\epsilon_{1}\right) \xi} F\left(\rho_{1}+\epsilon_{1}, c\right)\left[M_{2}-\frac{2 \beta\left(1+2 M_{3}\right)}{-S_{1} F\left(\rho_{1}+\epsilon_{1}, c\right)}\right] \geq 0 .
\end{aligned}
$$

If $\xi>\xi_{3}$, then $I_{-}(\xi)=0$ and inequality 4.8 holds immediately. The proof of this lemma is finished.

Now we introduce a non-empty, bounded, closed and convex subset of $C\left([-X, X], \mathbb{R}^{3}\right)$

$$
\begin{aligned}
\Omega_{X}:=\left\{(\phi, \varphi, \chi)(\xi) \in C\left([-X, X], \mathbb{R}^{3}\right) \mid\right. & \phi(-X)=S_{-}(-X), \varphi(-X)=I_{-}(-X), \\
& \chi(-X)=R_{-}(-X), S_{-}(\xi) \leq \phi(\xi) \leq S_{+}(\xi), \\
& I_{-}(\xi) \leq \varphi(\xi) \leq I_{+}(\xi), R_{-}(\xi) \leq \chi(\xi) \leq R_{+}(\xi) \\
& \text { for any } \xi \in[-X, X]\},
\end{aligned}
$$

which is endowed with the usual supremum norm, where $X \gg l:=\max \left\{\left|\xi_{3}\right|, c \tau, 1\right\}$. On a closed interval $[-X-l, X+l]$, we construct the following nonnegative continuous functions

$$
\widehat{\phi}(\xi):=\left\{\begin{array}{ll}
S_{-}(\xi), & \xi \in I_{1}, \\
\phi(\xi), & \xi \in I_{2}, \\
\phi(X), & \xi \in I_{3},
\end{array} \quad \widehat{\varphi}(\xi):=\left\{\begin{array}{ll}
I_{-}(\xi), & \xi \in I_{1}, \\
\varphi(\xi), & \xi \in I_{2}, \\
\varphi(X), & \xi \in I_{3},
\end{array} \quad \widehat{\chi}(\xi):= \begin{cases}R_{-}(\xi), & \xi \in I_{1}, \\
\chi(\xi), & \xi \in I_{2}, \\
\chi(X), & \xi \in I_{3},\end{cases}\right.\right.
$$

where $I_{1}=[-X-l,-X], I_{2}=(-X, X), I_{3}=[X, X+l]$ and $(\phi, \varphi, \chi)(\xi) \in \Omega_{X}$. For any $\xi \in[-X-l, X+l]$, one can check that

$$
S_{-}(\xi) \leq \widehat{\phi}(\xi) \leq S_{+}(\xi), \quad I_{-}(\xi) \leq \widehat{\varphi}(\xi) \leq I_{+}(\xi) \quad \text { and } \quad R_{-}(\xi) \leq \widehat{\chi}(\xi) \leq R_{+}(\xi)
$$


Consider an initial value problem

$$
\left\{\begin{array}{l}
c S^{\prime}(\xi)=d_{s}[\widehat{\phi}(\xi+1)+\widehat{\phi}(\xi-1)-2 S(\xi)]-\alpha S(\xi)+\alpha \phi(\xi)-\frac{\beta \phi(\xi) \widehat{\varphi}(\xi-c \tau)}{\phi(\xi)+\widehat{\varphi}(\xi-c \tau)+\chi(\xi)}, \\
c I^{\prime}(\xi)=d_{i}[\widehat{\varphi}(\xi+1)+\widehat{\varphi}(\xi-1)-2 I(\xi)]+\frac{\beta \phi(\xi) \widehat{\varphi}(\xi-c \tau)}{\phi(\xi)+\widehat{\varphi}(\xi-c \tau)+\chi(\xi)}-(\gamma+\delta) I(\xi), \\
c R^{\prime}(\xi)=d_{r}[\widehat{\chi}(\xi+1)+\widehat{\chi}(\xi-1)-2 R(\xi)]+\gamma \varphi(\xi), \\
S(-X)=S_{-}(-X), \quad I(-X)=I_{-}(-X), \quad R(-X)=R_{-}(-X),
\end{array}\right.
$$

where $\xi \in[-X, X]$ and the constant $\alpha>\beta$. The general theory of ordinary differential equations guarantees that initial problem (4.14) has a unique solution $\left(S_{X}, I_{X}, R_{X}\right)(\xi) \in$ $C^{1}\left([-X, X], \mathbb{R}^{3}\right)$. Also the solution of $(4.14)$ can be written by the following integral form

$$
\left\{\begin{array}{l}
S_{X}(\xi)=e^{-\frac{2 d_{s}+\alpha}{c}(\xi+X)} S_{-}(-X)+\frac{1}{c} \int_{-X}^{\xi} e^{\frac{2 d_{s}+\alpha}{c}(\eta-\xi)} H_{1}(\phi, \varphi, \chi)(\eta) d \eta, \\
I_{X}(\xi)=e^{-\frac{2 d_{i}+\gamma+\delta}{c}(\xi+X)} I_{-}(-X)+\frac{1}{c} \int_{-X}^{\xi} e^{\frac{2 d_{i}+\gamma+\delta}{c}(\eta-\xi)} H_{2}(\phi, \varphi, \chi)(\eta) d \eta, \\
R_{X}(\xi)=\frac{1}{c} \int_{-X}^{\xi} e^{\frac{2 d_{r}}{c}(\eta-\xi)} H_{3}(\phi, \varphi, \chi)(\eta) d \eta,
\end{array}\right.
$$

where

$$
\begin{aligned}
& H_{1}(\phi, \varphi, \chi)(\eta)=d_{s} \widehat{\phi}(\eta+1)+d_{s} \widehat{\phi}(\eta-1)+\alpha \phi(\eta)-\frac{\beta \phi(\eta) \widehat{\varphi}(\eta-c \tau)}{\phi(\eta)+\widehat{\varphi}(\eta-c \tau)+\chi(\eta)}, \\
& H_{2}(\phi, \varphi, \chi)(\eta)=d_{i} \widehat{\varphi}(\eta+1)+d_{i} \widehat{\varphi}(\eta-1)+\frac{\beta \phi(\eta) \widehat{\varphi}(\eta-c \tau)}{\phi(\eta)+\widehat{\varphi}(\eta-c \tau)+\chi(\eta)}, \\
& H_{3}(\phi, \varphi, \chi)(\eta)=d_{r} \widehat{\chi}(\eta+1)+d_{r} \widehat{\chi}(\eta-1)+\gamma \varphi(\eta) .
\end{aligned}
$$

Note that since $\alpha>\beta, H_{1}(\phi, \varphi, \chi)$ is decreasing with respect to $\varphi$ and is increasing in both $\phi$ and $\chi ; H_{2}(\phi, \varphi, \chi)$ is decreasing with respect to $\chi$ and increasing in both $\phi$ and $\varphi$; $H_{3}(\phi, \varphi, \chi)$ is increasing in both $\varphi$ and $\chi$.

By 4.15 we define a nonlinear operator $\mathcal{O}=\left(\mathcal{O}_{1}, \mathcal{O}_{2}, \mathcal{O}_{3}\right): \Omega_{X} \mapsto C^{1}\left([-X, X], \mathbb{R}^{3}\right)$ as follows.

$$
\mathcal{O}_{1}(\phi, \varphi, \chi)(\xi):=S_{X}(\xi), \quad \mathcal{O}_{2}(\phi, \varphi, \chi)(\xi):=I_{X}(\xi) \quad \text { and } \quad \mathcal{O}_{3}(\phi, \varphi, \chi)(\xi):=R_{X}(\xi)
$$

In the next two lemmas, we will prove that the operator $\mathcal{O}$ is completely continuous which maps $\Omega_{X}$ into itself.

Lemma 4.2. The operator $\mathcal{O}$ satisfies $\mathcal{O}\left(\Omega_{X}\right) \subseteq \Omega_{X}$.

Proof. For any $(\phi, \varphi, \chi)(\xi) \in \Omega_{X}$, we only need to show that

$$
S_{-}(\xi) \leq \mathcal{O}_{1}(\phi, \varphi, \chi)(\xi) \leq S_{+}(\xi), \quad I_{-}(\xi) \leq \mathcal{O}_{2}(\phi, \varphi, \chi)(\xi) \leq I_{+}(\xi)
$$

and

$$
R_{-}(\xi) \leq \mathcal{O}_{3}(\phi, \varphi, \chi)(\xi) \leq R_{+}(\xi) \quad \text { for } \xi \in[-X, X]
$$


By the monotonicity of $H_{1}(\phi, \varphi, \chi)$, we obtain from (4.4), 4.7) and 4.13 that

$$
\begin{aligned}
& d_{s}\left[\widehat{\phi}(\xi+1)+\widehat{\phi}(\xi-1)-2 S_{+}(\xi)\right]-c S_{+}^{\prime}(\xi)-\alpha S_{+}(\xi)+\alpha \phi(\xi) \\
& -\frac{\beta \phi(\xi) \widehat{\varphi}(\xi-c \tau)}{\phi(\xi)+\widehat{\varphi}(\xi-c \tau)+\chi(\xi)} \\
\leq & d_{s} D\left[S_{+}\right](\xi)-c S_{+}^{\prime}(\xi)-\alpha S_{+}(\xi)+\alpha S_{+}(\xi)-\frac{\beta S_{+}(\xi) I_{-}(\xi-c \tau)}{S_{+}(\xi)+I_{-}(\xi-c \tau)+R_{+}(\xi)} \\
\leq & 0 \quad \text { for } \xi \in[-X, X]
\end{aligned}
$$

and

$$
\begin{aligned}
& d_{s}\left[\widehat{\phi}(\xi+1)+\widehat{\phi}(\xi-1)-2 S_{-}(\xi)\right]-c S_{-}^{\prime}(\xi)-\alpha S_{-}(\xi)+\alpha \phi(\xi) \\
& -\frac{\beta \phi(\xi) \widehat{\varphi}(\xi-c \tau)}{\phi(\xi)+\widehat{\varphi}(\xi-c \tau)+\chi(\xi)} \\
\geq & d_{s} D\left[S_{-}\right](\xi)-c S_{-}^{\prime}(\xi)-\alpha S_{-}(\xi)+\alpha S_{-}(\xi)-\frac{\beta S_{-}(\xi) I_{+}(\xi-c \tau)}{S_{-}(\xi)+I_{+}(\xi-c \tau)+R_{-}(\xi)} \\
\geq & 0 \quad \text { for } \xi \in\left[-X, \xi_{1}\right) \cup\left(\xi_{1}, X\right] .
\end{aligned}
$$

Inequalities (4.16) and (4.17) are equivalent to

$$
c S_{+}^{\prime}(\xi)+\left(2 d_{s}+\alpha\right) S_{+}(\xi) \geq H_{1}(\phi, \varphi, \chi)(\xi) \quad \text { for } \xi \in[-X, X]
$$

and

$$
c S_{-}^{\prime}(\xi)+\left(2 d_{s}+\alpha\right) S_{-}(\xi) \leq H_{1}(\phi, \varphi, \chi)(\xi) \quad \text { for } \xi \in\left[-X, \xi_{1}\right) \cup\left(\xi_{1}, X\right],
$$

which imply that

$$
\begin{aligned}
S_{+}(\xi) & \geq e^{-\frac{2 d_{s}+\alpha}{c}(\xi+X)} S_{+}(-X)+\frac{1}{c} \int_{-X}^{\xi} e^{\frac{2 d_{s}+\alpha}{c}(\eta-\xi)} H_{1}(\phi, \varphi, \chi)(\eta) d \eta \\
& \geq e^{-\frac{2 d_{s}+\alpha}{c}(\xi+X)} S_{-}(-X)+\frac{1}{c} \int_{-X}^{\xi} e^{\frac{2 d_{s}+\alpha}{c}(\eta-\xi)} H_{1}(\phi, \varphi, \chi)(\eta) d \eta \\
& =\mathcal{O}_{1}(\phi, \varphi, \chi)(\xi) \quad \text { for } \xi \in[-X, X]
\end{aligned}
$$

and

$$
\begin{aligned}
S_{-}(\xi) & \leq e^{-\frac{2 d_{s}+\alpha}{c}(\xi+X)} S_{-}(-X)+\frac{1}{c} \int_{-X}^{\xi} e^{\frac{2 d_{s}+\alpha}{c}(\eta-\xi)} H_{1}(\phi, \varphi, \chi)(\eta) d \eta \\
& =\mathcal{O}_{1}(\phi, \varphi, \chi)(\xi) \quad \text { for } \xi \in\left[-X, \xi_{1}\right) \cup\left(\xi_{1}, X\right] .
\end{aligned}
$$

In analogous manners, one can have that

$$
\begin{array}{ll}
I_{+}(\xi) \geq \mathcal{O}_{2}(\phi, \varphi, \chi)(\xi) & \text { for } \xi \in\left[-X, \xi_{2}\right) \cup\left(\xi_{2}, X\right], \\
I_{-}(\xi) \leq \mathcal{O}_{2}(\phi, \varphi, \chi)(\xi) & \text { for } \xi \in\left[-X, \xi_{3}\right) \cup\left(\xi_{3}, X\right]
\end{array}
$$


and

$$
R_{-}(\xi) \leq \mathcal{O}_{3}(\phi, \varphi, \chi)(\xi) \leq R_{+}(\xi) \text { for } \xi \in[-X, X]
$$

By the continuity of $\left(S_{ \pm}, I_{ \pm}, R_{ \pm}\right)(\xi)$ and the operator $\mathcal{O}$ on $[-X, X]$, we complete the proof.

Lemma 4.3. The operator $\mathcal{O}$ is completely continuous with respect to the supremum norm in $C\left([-X, X], \mathbb{R}^{3}\right)$.

Proof. Since $\left(S_{X}, I_{X}, R_{X}\right)(\xi) \in C^{1}\left([-X, X], \mathbb{R}^{3}\right)$ satisfies 4.15$)$, for $(\phi, \varphi, \chi)(\xi) \in \Omega_{X}$, we get that $S_{X}^{\prime}(\xi), I_{X}^{\prime}(\xi)$ and $R_{X}^{\prime}(\xi)$ are bounded on $[-X, X]$. Then applying Arzela-Ascoli theorem gives that the operator $\mathcal{O}$ is compact.

For any $\Phi_{i}(\xi):=\left(\phi_{i}, \varphi_{i}, \chi_{i}\right)(\xi) \in \Omega_{X}, i=1,2$, we deduce that

$$
\begin{aligned}
& \left|H_{1}\left(\phi_{1}, \varphi_{1}, \chi_{1}\right)(\xi)-H_{1}\left(\phi_{2}, \varphi_{2}, \chi_{2}\right)(\xi)\right| \\
\leq & d_{s}\left|\widehat{\phi}_{1}(\xi+1)-\widehat{\phi}_{2}(\xi+1)+\widehat{\phi}_{1}(\xi-1)-\widehat{\phi}_{2}(\xi-1)\right|+\alpha\left|\phi_{1}(\xi)-\phi_{2}(\xi)\right| \\
& +\left|\frac{\beta \phi_{1}(\xi) \widehat{\varphi}_{1}(\xi-c \tau)}{\phi_{1}(\xi)+\widehat{\varphi}_{1}(\xi-c \tau)+\chi_{1}(\xi)}-\frac{\beta \phi_{2}(\xi) \widehat{\varphi}_{2}(\xi-c \tau)}{\phi_{2}(\xi)+\widehat{\varphi}_{2}(\xi-c \tau)+\chi_{2}(\xi)}\right| \\
\leq & 2 d_{s} \sup _{\xi \in[-X, X]}\left|\phi_{1}(\xi)-\phi_{2}(\xi)\right|+\alpha \sup _{\xi \in[-X, X]}\left|\phi_{1}(\xi)-\phi_{2}(\xi)\right|+2 \beta \sup _{\xi \in[-X, X]}\left|\phi_{1}(\xi)-\phi_{2}(\xi)\right| \\
& +2 \beta \sup _{\xi \in[-X, X]}\left|\varphi_{1}(\xi)-\varphi_{2}(\xi)\right|+\beta \sup _{\xi \in[-X, X]}\left|\chi_{1}(\xi)-\chi_{2}(\xi)\right| \\
\leq & \left(2 d_{s}+\alpha+5 \beta\right) \sup _{\xi \in[-X, X]}\left|\Phi_{1}(\xi)-\Phi_{2}(\xi)\right| .
\end{aligned}
$$

Then we derive that

$$
\begin{aligned}
& \left|\mathcal{O}_{1}\left(\phi_{1}, \varphi_{1}, \chi_{1}\right)(\xi)-\mathcal{O}_{1}\left(\phi_{2}, \varphi_{2}, \chi_{2}\right)(\xi)\right| \\
\leq & \frac{1}{c} \int_{-X}^{\xi} e^{\frac{2 d_{s}+\alpha}{c}(\eta-\xi)}\left|H_{1}\left(\phi_{1}, \varphi_{1}, \chi_{1}\right)(\eta)-H_{1}\left(\phi_{2}, \varphi_{2}, \chi_{2}\right)(\eta)\right| d \eta \\
\leq & \frac{2 d_{s}+\alpha+5 \beta}{c} \sup _{\xi \in[-X, X]}\left|\Phi_{1}(\xi)-\Phi_{2}(\xi)\right| \int_{-X}^{\xi} e^{\frac{2 d_{s}+\alpha}{c}(\eta-\xi)} d \eta \\
\leq & \left(1+\frac{5 \beta}{2 d_{s}+\alpha}\right) \sup _{\xi \in[-X, X]}\left|\Phi_{1}(\xi)-\Phi_{2}(\xi)\right| .
\end{aligned}
$$

Similarly, one can obtain that

$$
\left|\mathcal{O}_{j}\left(\phi_{1}, \varphi_{1}, \chi_{1}\right)(\xi)-\mathcal{O}_{j}\left(\phi_{2}, \varphi_{2}, \chi_{2}\right)(\xi)\right| \leq C \sup _{\xi \in[-X, X]}\left|\Phi_{1}(\xi)-\Phi_{2}(\xi)\right| \quad \text { for } j=2,3
$$

where the positive constant $C$ depends on $d_{i}, d_{r}, \beta, \gamma$ and $\delta$. This ends the proof. 
Applying Lemmas 4.2, 4.3 and Schauder's fixed point theorem yields that the operator $\mathcal{O}$ has a fixed point, which is a solution of the system

$$
\left\{\begin{array}{l}
c S_{X}^{\prime}(\xi)=d_{s} D\left[S_{X}\right](\xi)-\frac{\beta S_{X}(\xi) I_{X}(\xi-c \tau)}{S_{X}(\xi)+I_{X}(\xi-c \tau)+R_{X}(\xi)} \\
c I_{X}^{\prime}(\xi)=d_{i} D\left[I_{X}\right](\xi)+\frac{\beta S_{X}(\xi) I_{X}(\xi-c \tau)}{S_{X}(\xi)+I_{X}(\xi-c \tau)+R_{X}(\xi)}-(\gamma+\delta) I_{X}(\xi) \\
c R_{X}^{\prime}(\xi)=d_{r} D\left[R_{X}\right](\xi)+\gamma I_{X}(\xi)
\end{array}\right.
$$

such that

$$
S_{-}(\xi) \leq S_{X}(\xi) \leq S_{+}(\xi), \quad I_{-}(\xi) \leq I_{X}(\xi) \leq I_{+}(\xi)
$$

and

$$
R_{-}(\xi) \leq R_{X}(\xi) \leq R_{+}(\xi) \text { for } \xi \in[-X+l, X-l]
$$

Lemma 4.4. If $R_{0}>1$ and $c>c^{*}$, then system 1.2 admits a solution $(S, I, R)(\xi)$ on $\mathbb{R}$ such that

$$
S_{-}(\xi) \leq S(\xi) \leq S_{+}(\xi), \quad I_{-}(\xi) \leq I(\xi) \leq I_{+}(\xi) \quad \text { and } \quad R_{-}(\xi) \leq R(\xi) \leq R_{+}(\xi) .
$$

Proof. Select an increasing sequence $\left\{X_{n}\right\}_{n \in \mathbb{N}}$ satisfying $X_{n} \gg l$ for each $n$ and $X_{n} \rightarrow+\infty$ as $n \rightarrow \infty$. Let $\left(S_{X_{n}}, I_{X_{n}}, R_{X_{n}}\right)(\xi), n \in \mathbb{N}$, be the solution of 4.18 with $X=X_{n}$. For any fixed $N \in \mathbb{N}$, since $R_{+}(\xi)$ is bounded on $\left[-X_{N}, X_{N}\right]$, we have that the sequences $\left\{S_{X_{n}}(\xi)\right\}_{n \geq N},\left\{I_{X_{n}}(\xi)\right\}_{n \geq N},\left\{R_{X_{n}}(\xi)\right\}_{n \geq N}$ and $\left\{\frac{S_{X_{n}}(\xi) I_{X_{n}}(\xi-c \tau)}{S_{X_{n}}(\xi)+I_{X_{n}}(\xi-c \tau)+R_{X_{n}}(\xi)}\right\}_{n \geq N}$ are uniformly bounded on $\left[-X_{N}, X_{N}\right]$. By (4.18) with $X=X_{n}$, we get that the sequences

$$
\left\{S_{X_{n}}^{\prime}(\xi)\right\}_{n \geq N}, \quad\left\{I_{X_{n}}^{\prime}(\xi)\right\}_{n \geq N} \quad \text { and } \quad\left\{R_{X_{n}}^{\prime}(\xi)\right\}_{n \geq N}
$$

are uniformly bounded on $\left[-X_{N}+l, X_{N}-l\right]$. Differentiating 4.18 with $X=X_{n}$, we obtain that the sequences

$$
\left\{S_{X_{n}}^{\prime \prime}(\xi)\right\}_{n \geq N}, \quad\left\{I_{X_{n}}^{\prime \prime}(\xi)\right\}_{n \geq N} \quad \text { and } \quad\left\{R_{X_{n}}^{\prime \prime}(\xi)\right\}_{n \geq N}
$$

are uniformly bounded on $\left[-X_{N}+2 l, X_{N}-2 l\right]$. Utilizing Arzela-Ascoli theorem and a standard diagonal extraction argument, we deduce that there is a subsequence which is still denoted by $\left(S_{X_{n}}, I_{X_{n}}, R_{X_{n}}\right)(\xi)$ such that

$$
S_{X_{n}}(\xi) \rightarrow S(\xi), \quad I_{X_{n}}(\xi) \rightarrow I(\xi), \quad R_{X_{n}}(\xi) \rightarrow R(\xi) \quad \text { in } C_{\text {loc }}^{1}(\mathbb{R}) \text { as } n \rightarrow \infty
$$

Moreover, there holds

$$
S_{-}(\xi) \leq S(\xi) \leq S_{+}(\xi), \quad I_{-}(\xi) \leq I(\xi) \leq I_{+}(\xi) \text { and } R_{-}(\xi) \leq R(\xi) \leq R_{+}(\xi) \text { for } \xi \in \mathbb{R}
$$

The proof of this lemma is finished. 
Based on Lemma 4.4 , we will proving the following result.

Theorem 4.5. For a given constant $S_{1}>0$, if $R_{0}>1$ and $c>c^{*}$, then system (1.1) has a nontrivial positive traveling wave solution $(S, I, R)(\xi)$ satisfying

(i) $0<S(\xi)<S_{1}, 0<I(\xi)<\left(R_{0}-1\right) S_{1}$ and $R(\xi)>0$ for $\xi \in \mathbb{R}$.

(ii) $(S, I, R)(-\infty)=\left(S_{1}, 0,0\right)$. If $\xi \rightarrow-\infty$, then $I(\xi)=O\left(e^{\rho_{1} \xi}\right)$.

(iii) $I(+\infty)=0, S(+\infty):=S_{2}$ exists and $S_{2}<S_{1}$.

(iv) $(\gamma+\delta) \int_{\mathbb{R}} I(\xi) d \xi=\beta \int_{\mathbb{R}} \frac{S(\xi) I(\xi-c \tau)}{S(\xi)+I(\xi-c \tau)+R(\xi)} d \xi=c\left(S_{1}-S_{2}\right)$.

(v) If $\lim \sup _{\xi \rightarrow+\infty} R(\xi)<+\infty$, then $R(+\infty)=\gamma\left(S_{1}-S_{2}\right) /(\gamma+\delta)$ and $S^{\prime}(\xi), I^{\prime}(\xi), R^{\prime}(\xi)$ $\rightarrow 0$ as $\xi \rightarrow \pm \infty$.

Proof. (i) By (4.19), we have that $S(\xi)>0$ on $\mathbb{R}$. Suppose that $I\left(\widetilde{\xi}_{1}\right)=0$ for some $\widetilde{\xi}_{1} \in \mathbb{R}$, then $I^{\prime}\left(\widetilde{\xi}_{1}\right)=0$. It follows from the second equation in 1.2 that

$$
I\left(\widetilde{\xi}_{1}+1\right)=I\left(\widetilde{\xi}_{1}-1\right)=0 .
$$

By induction we obtain that

$$
I\left(\widetilde{\xi}_{1}-n\right)=0 \quad \text { for } n \in \mathbb{Z}
$$

which contradicts that $I(\xi) \geq I_{-}(\xi)>0$ for $\xi \in\left(-\infty, \xi_{3}\right)$. Hence $I(\xi)>0$ on $\mathbb{R}$. Assume that $R\left(\widetilde{\xi}_{2}\right)=0$ for some $\widetilde{\xi}_{2} \in \mathbb{R}$, then $R^{\prime}\left(\widetilde{\xi}_{2}\right)=0$. From the third equation in 1.2 , we deduce that $I\left(\widetilde{\xi}_{2}\right)=0$, a contradiction appears. Thus $R(\xi)>0$ on $\mathbb{R}$. Suppose that $S\left(\widetilde{\xi}_{3}\right)=S_{1}$ for some $\widetilde{\xi}_{3} \in \mathbb{R}$. Then $S^{\prime}\left(\widetilde{\xi}_{3}\right)=0$. By the first equation in $(1.2)$, we have that

$$
\begin{aligned}
0 & =d_{s} D[S]\left(\widetilde{\xi}_{3}\right)-c S^{\prime}\left(\widetilde{\xi}_{3}\right)-\frac{\beta S\left(\widetilde{\xi}_{3}\right) I\left(\widetilde{\xi}_{3}-c \tau\right)}{S\left(\widetilde{\xi}_{3}\right)+I\left(\widetilde{\xi}_{3}-c \tau\right)+R\left(\widetilde{\xi}_{3}\right)} \\
& \leq-\frac{\beta S_{1} I\left(\widetilde{\xi}_{3}-c \tau\right)}{S_{1}+I\left(\widetilde{\xi}_{3}-c \tau\right)+R\left(\widetilde{\xi}_{3}\right)}<0,
\end{aligned}
$$

which yields a contradiction. So $S(\xi)<S_{1}$ on $\mathbb{R}$. Assume that $I\left(\widetilde{\xi}_{4}\right)=\left(R_{0}-1\right) S_{1}$ for some $\widetilde{\xi}_{4} \in \mathbb{R}$. Then $I^{\prime}\left(\widetilde{\xi}_{4}\right)=0$. Using the second equation in $(1.2)$, we get that

$$
\begin{aligned}
0 & =d_{i} D[I]\left(\widetilde{\xi}_{4}\right)-c I^{\prime}\left(\widetilde{\xi}_{4}\right)+\frac{\beta S\left(\widetilde{\xi}_{4}\right) I\left(\widetilde{\xi}_{4}-c \tau\right)}{S\left(\widetilde{\xi}_{4}\right)+I\left(\widetilde{\xi}_{4}-c \tau\right)+R\left(\widetilde{\xi}_{4}\right)}-(\gamma+\delta) I\left(\widetilde{\xi}_{4}\right) \\
& <\frac{\beta S_{1}\left(R_{0}-1\right) S_{1}}{S_{1}+\left(R_{0}-1\right) S_{1}}-(\gamma+\delta)\left(R_{0}-1\right) S_{1}=0
\end{aligned}
$$

which leads to a contradiction. Therefore, $I(\xi)<\left(R_{0}-1\right) S_{1}$ on $\mathbb{R}$. 
(ii) Applying squeeze rule in 4.19 yields that

$$
(S, I, R)(-\infty)=\left(S_{1}, 0,0\right) \quad \text { and } \quad I(\xi)=O\left(e^{\rho_{1} \xi}\right) \quad \text { as } \xi \rightarrow-\infty
$$

(iii) We claim that $I(\xi)$ is integrable on $\mathbb{R}$. Integrating the first equation in 1.2 over $[x, y]$ yields

$$
\begin{aligned}
& \int_{x}^{y} \frac{\beta S(\xi) I(\xi-c \tau)}{S(\xi)+I(\xi-c \tau)+R(\xi)} d \xi \\
= & d_{s} \int_{x}^{y} D[S](\xi)-c \int_{x}^{y} S^{\prime}(\xi) d \xi \\
= & d_{s} \int_{x}^{y} \int_{0}^{1} S^{\prime}(\xi+\theta) d \theta d \xi-d_{s} \int_{x}^{y} \int_{0}^{1} S^{\prime}(\xi-\theta) d \theta d \xi-c S(y)+c S(x) \\
= & d_{s} \int_{0}^{1}[S(y+\theta)-S(x+\theta)] d \theta+d_{s} \int_{0}^{1}[S(x-\theta)-S(y-\theta)] d \theta-c S(y)+c S(x) \\
< & \left(2 d_{s}+c\right) S_{1} \quad\left(\text { since } 0<S(\xi)<S_{1} \text { on } \mathbb{R}\right),
\end{aligned}
$$

which together with the positiveness of $S(\xi), I(\xi)$ and $R(\xi)$ on $\mathbb{R}$ implies

$$
\int_{\mathbb{R}} \frac{S(\xi) I(\xi-c \tau)}{S(\xi)+I(\xi-c \tau)+R(\xi)} d \xi<+\infty .
$$

Note that $0<I(\xi)<\left(R_{0}-1\right) S_{0}$ for $\xi \in \mathbb{R}$. Then an integration of the second equation in 1.2 over $[\eta, \zeta]$ gives

$$
\begin{aligned}
& (\gamma+\delta) \int_{\eta}^{\zeta} I(\xi) d \xi \\
= & d_{i} \int_{\eta}^{\zeta} D[I](\xi)-c \int_{\eta}^{\zeta} I^{\prime}(\xi) d \xi+\int_{\eta}^{\zeta} \frac{\beta S(\xi) I(\xi-c \tau)}{S(\xi)+I(\xi-c \tau)+R(\xi)} d \xi \\
< & d_{i} \int_{\eta}^{\zeta} \int_{0}^{1} I^{\prime}(\xi+\theta) d \theta d \xi-d_{i} \int_{\eta}^{\zeta} \int_{0}^{1} I^{\prime}(\xi-\theta) d \theta d \xi-c I(\zeta)+c I(\eta)+\left(2 d_{s}+c\right) S_{1} \\
= & d_{i} \int_{0}^{1}[I(\zeta+\theta)-I(\eta+\theta)] d \theta+d_{i} \int_{0}^{1}[I(\eta-\theta)-I(\zeta-\theta)] d \theta \\
& -c I(\zeta)+c I(\eta)+\left(2 d_{s}+c\right) S_{1} \\
< & \left(2 d_{i}+c\right)\left(R_{0}-1\right) S_{0}+\left(2 d_{s}+c\right) S_{1} .
\end{aligned}
$$

This together with the positiveness of $I(\xi)$ on $\mathbb{R}$ ensures that

$$
\int_{-\infty}^{\infty} I(\xi) d \xi<+\infty
$$

Recall that $0<S(\xi)<S_{1}, 0<I(\xi)<\left(R_{0}-1\right) S_{1}$ and $R(\xi)>0$ for $\xi \in \mathbb{R}$. Then it follows from the second equation in 1.2 that $I^{\prime}(\xi)$ is uniformly bounded on $\mathbb{R}$. Hence 
$I(+\infty)=0$. Now we investigate the existence of $S(+\infty)$. Assume for the contrary that $\lim \sup _{\xi \rightarrow+\infty} S(\xi)>\liminf _{\xi \rightarrow+\infty} S(\xi)$. Then applying Fluctuation Lemma 31, Lemma 2.2] yields that there are two sequences $\left\{\xi_{n}\right\}_{n \in \mathbb{N}}$ and $\left\{\eta_{n}\right\}_{n \in \mathbb{N}}$ satisfying $\xi_{n}, \eta_{n} \rightarrow$ $+\infty$ as $n \rightarrow \infty$ such that

(4.20) $\lim _{n \rightarrow \infty} S\left(\xi_{n}\right)=\limsup _{\xi \rightarrow+\infty} S(\xi):=m_{1} \quad$ and $\quad \lim _{n \rightarrow \infty} S\left(\eta_{n}\right)=\liminf _{\xi \rightarrow+\infty} S(\xi):=m_{2}<m_{1}$.

Denote

$$
S_{n}(y):=S\left(\eta_{n}+y\right), \quad I_{n}(y):=I\left(\eta_{n}+y\right) \quad \text { and } \quad R_{n}(y):=R\left(\eta_{n}+y\right), \quad y \in \mathbb{R} .
$$

We infer from $I(+\infty)=0$ that $I_{n}(y) \rightarrow 0$ in $C_{\text {loc }}(\mathbb{R})$ as $n \rightarrow \infty$. By 4.19) and the first equation in 1.2 , we deduce that $S(\xi), S^{\prime}(\xi)$ and $S^{\prime \prime}(\xi)$ are uniformly bounded on $\mathbb{R}$. Then there exists a subsequence $\left\{n_{k}\right\}$ by a diagonal extraction process, which is still denoted by $\{n\}$, such that $S_{n}(y) \rightarrow S_{\infty}(y)$ in $C_{\text {loc }}^{1}(\mathbb{R})$ as $n \rightarrow \infty$. Note that $S_{\infty}(0)=m_{2}$. From 4.20 and the first equation in 1.2 , we have that

$$
c S_{n}^{\prime}(y)=d_{s} D\left[S_{n}\right](y)-\frac{\beta S_{n}(y) I_{n}(y-c \tau)}{S_{n}(y)+I_{n}(y-c \tau)+R_{n}(y)}, \quad y \in \mathbb{R} .
$$

Passing to the limits in 4.21 as $n \rightarrow \infty$ gives that

$$
c S_{\infty}^{\prime}(y)=d_{s} D\left[S_{\infty}\right](y), \quad y \in \mathbb{R} .
$$

With the aid of [4, Theorem 3.1 and Remark 3.1], we get from 4.22 that

$$
S_{\infty}(y)=a_{1}+a_{2} e^{\nu y}, \quad y \in \mathbb{R}
$$

where $a_{1}, a_{2}$ are constants and $\nu$ is the unique positive root of $d_{s}\left(e^{\nu}+e^{-\nu}-2\right)-c \nu=0$. Then by the boundedness of $S_{\infty}(y)$ on $\mathbb{R}$ and (4.23), we derive that $a_{2}=0$. Thus $S_{\infty}(y)=a_{1}=S_{\infty}(0)=m_{2}$, which ensures that

$$
\lim _{n \rightarrow \infty} S\left(\eta_{n}+y\right)=m_{2} \quad \text { in } C_{\text {loc }}^{1}(\mathbb{R}) .
$$

Analogously,

$$
\lim _{n \rightarrow \infty} S\left(\xi_{n}+y\right)=m_{1} \quad \text { in } C_{\mathrm{loc}}^{1}(\mathbb{R}) .
$$

By the similar arguments as that in Lemma 2.4, one can deduce that

$$
\int_{\mathbb{R}} \frac{S(\xi) I(\xi-c \tau)}{S(\xi)+I(\xi-c \tau)+R(\xi)} d \xi<+\infty,
$$

which leads to

$$
\lim _{n \rightarrow \infty} \int_{\eta_{n}}^{\xi_{n}} \frac{S(\xi) I(\xi-c \tau)}{S(\xi)+I(\xi-c \tau)+R(\xi)} d \xi=0
$$


Integrating the first equation in 1.2 over $\left[\eta_{n}, \xi_{n}\right]$ and using 4.24 - 4.26 and dominated convergence theorem, we obtain that

$$
\begin{aligned}
0< & c\left(m_{1}-m_{2}\right) \\
= & c \lim _{n \rightarrow \infty}\left[S\left(\xi_{n}\right)-S\left(\eta_{n}\right)\right] \\
= & d_{s} \lim _{n \rightarrow \infty} \int_{\eta_{n}}^{\xi_{n}}[S(\xi+1)-S(\xi)] d \xi+d_{s} \lim _{n \rightarrow \infty} \int_{\eta_{n}}^{\xi_{n}}[S(\xi-1)-S(\xi)] d \xi \\
& -\lim _{n \rightarrow \infty} \int_{\eta_{n}}^{\xi_{n}} \frac{\beta S(\xi) I(\xi-c \tau)}{S(\xi)+I(\xi-c \tau)+R(\xi)} d \xi \\
= & d_{s} \lim _{n \rightarrow \infty} \int_{\eta_{n}}^{\xi_{n}} \int_{0}^{1} S^{\prime}(\xi+\theta) d \theta d \xi-d_{s} \lim _{n \rightarrow \infty} \int_{\eta_{n}}^{\xi_{n}} \int_{0}^{1} S^{\prime}(\xi-\theta) d \theta d \xi \\
= & d_{s} \lim _{n \rightarrow \infty} \int_{0}^{1}\left[S\left(\xi_{n}+\theta\right)-S\left(\eta_{n}+\theta\right)\right] d \theta-d_{s} \lim _{n \rightarrow \infty} \int_{0}^{1}\left[S\left(\xi_{n}-\theta\right)-S\left(\eta_{n}-\theta\right)\right] d \theta \\
= & 0 .
\end{aligned}
$$

A contradiction appears. Hence $S(+\infty)$ exists and we denote it by $S_{2}$. Then we present $S_{2}<S_{1}$. Since $S(\xi)<S_{1}$ on $\mathbb{R}$, we have $S_{2} \leq S_{1}$. Assume that $S_{2}=S_{1}$. An integration of the first equation in 1.2 over $\mathbb{R}$ yields

$$
\int_{\mathbb{R}} \frac{S(\xi) I(\xi-c \tau)}{S(\xi)+I(\xi-c \tau)+R(\xi)} d \xi=0
$$

which contradicts that fact that

$$
\int_{\mathbb{R}} \frac{S(\xi) I(\xi-c \tau)}{S(\xi)+I(\xi-c \tau)+R(\xi)} d \xi>0
$$

Therefore, $S_{2}<S_{1}$.

(iv) Integrating the first two equations in 1.2 over $\mathbb{R}$, respectively, and using the asymptotic boundaries of $S(\xi)$ and $I(\xi)$, we have

$$
(\gamma+\delta) \int_{\mathbb{R}} I(\xi) d \xi=\beta \int_{\mathbb{R}} \frac{S(\xi) I(\xi-c \tau)}{S(\xi)+I(\xi-c \tau)+R(\xi)} d \xi=c\left(S_{1}-S_{2}\right)
$$

Suppose that $\lim \sup _{\xi \rightarrow+\infty} R(\xi)>\liminf _{\xi \rightarrow+\infty} R(\xi)$. Then there exist two sequences $\left\{\zeta_{n}\right\}$ and $\left\{\vartheta_{n}\right\}$ satisfying $\zeta_{n}, \vartheta_{n} \rightarrow+\infty$ as $n \rightarrow \infty$ such that

$$
\lim _{n \rightarrow \infty} R\left(\zeta_{n}\right)=\limsup _{\xi \rightarrow+\infty} R(\xi):=m_{3} \quad \text { and } \quad \lim _{n \rightarrow \infty} R\left(\vartheta_{n}\right)=\liminf _{\xi \rightarrow+\infty} R(\xi):=m_{4}<m_{3} .
$$

By the analogous arguments as that in (iii), one can obtain that

$$
\lim _{n \rightarrow \infty} R\left(\zeta_{n}+y\right)=m_{3} \quad \text { and } \quad \lim _{n \rightarrow \infty} R\left(\vartheta_{n}+y\right)=m_{4} \quad \text { in } C_{\mathrm{loc}}^{1}(\mathbb{R})
$$


Hence integrating the third equation in $(1.2)$ over $\left[\vartheta_{n}, \zeta_{n}\right]$ and taking $n \rightarrow \infty$, we get

$$
\begin{aligned}
0< & c\left(m_{3}-m_{4}\right) \\
= & c \lim _{n \rightarrow \infty}\left[R\left(\zeta_{n}\right)-R\left(\vartheta_{n}\right)\right] \\
= & d_{r} \lim _{n \rightarrow \infty} \int_{\vartheta_{n}}^{\zeta_{n}}[R(\xi+1)-R(\xi)] d \xi+d_{r} \lim _{n \rightarrow \infty} \int_{\vartheta_{n}}^{\zeta_{n}}[R(\xi-1)-R(\xi)] d \xi \\
& +\lim _{n \rightarrow \infty} \int_{\vartheta_{n}}^{\zeta_{n}} \gamma I(\xi) d \xi \\
= & d_{r} \lim _{n \rightarrow \infty} \int_{\vartheta_{n}}^{\zeta_{n}} \int_{0}^{1} R^{\prime}(\xi+\theta) d \theta d \xi-d_{r} \lim _{n \rightarrow \infty} \int_{\vartheta_{n}}^{\zeta_{n}} \int_{0}^{1} R^{\prime}(\xi-\theta) d \theta d \xi \\
= & d_{r} \lim _{n \rightarrow \infty} \int_{0}^{1}\left[R\left(\zeta_{n}+\theta\right)-R\left(\vartheta_{n}+\theta\right)\right] d \theta-d_{r} \lim _{n \rightarrow \infty} \int_{0}^{1}\left[R\left(\zeta_{n}-\theta\right)-R\left(\vartheta_{n}-\theta\right)\right] d \theta \\
= & 0 .
\end{aligned}
$$

This contradiction guarantees that the existence of $R(+\infty)$. Integrating the third equation in (1.2) and utilizing (4.27), we obtain that

$$
R(+\infty)=\frac{\gamma\left(S_{1}-S_{2}\right)}{\gamma+\delta}
$$

Passing to the limits in $(1.2)$ as $\xi \rightarrow \pm \infty$, respectively, and employing the asymptotic boundary of $(S, I, R)(\xi)$, we get

$$
S^{\prime}(\xi), I^{\prime}(\xi), R^{\prime}(\xi) \rightarrow 0 \quad \text { as } \xi \rightarrow \pm \infty .
$$

The proof is completed.

Remark 4.6. In Theorem 4.5(v), we make use of a condition $\lim \sup _{\xi \rightarrow+\infty} R(\xi)<+\infty$ to prove the existence of $R(+\infty)$. From the view of mathematical biology, this condition may fit reality. However, we do not obtain it by rigorous analysis. We leave it for future investigation.

\section{Existence of critical traveling waves}

In this section, we will prove the existence result under the conditions $R_{0}>1$ and $c=c^{*}$. For this, we set

$$
M_{4}:=\frac{\left(R_{0}-1\right) S_{1} \rho^{*} e^{\rho^{*}+1}}{\rho^{*}+1} \text { and } \xi_{5}:=-\frac{1}{\rho^{*}}-1,
$$

where $\rho^{*}>0$ is defined in Lemma 2.2 and $S_{1}>0$ is a given constant. Choose a sufficient small constant $\rho_{5} \in\left(0, \rho^{*}\right)$ and a suitable constant $\sigma_{2}>0$ such that $\rho_{5}^{-1} \gg S_{1}$ and

$$
S_{1}-\rho_{5}^{-1} e^{\rho_{5} \xi}=\sigma_{2} e^{-\frac{\beta}{c^{*} \xi}}
$$


has two negative roots and we select the bigger one as $\xi_{4}$. By the choice of $\rho_{5}$, one can deduce that

$\xi_{4}<\xi_{5} \quad$ and $\quad \rho_{5}^{-1} d_{s}\left(2-e^{\rho_{5}}-e^{-\rho_{5}}\right)+c^{*}+\beta M_{4}\left(\xi-c^{*} \tau\right) e^{\left(\rho^{*}-\rho_{5}\right) \xi-\rho^{*} c^{*} \tau} \geq 0 \quad$ for $\xi<\xi_{4}$.

We choose suitable constants $M_{6}>0$ and $\epsilon_{2} \in\left(0, \min \left\{\rho_{3}, \rho^{*}\right\}\right)$ such that

$-G\left(\epsilon_{2}, c^{*}\right)+\frac{\gamma\left(R_{0}-1\right) S_{1} e^{-\epsilon_{2} \xi_{5}}}{M_{6}} \leq 0 \quad$ and $\quad-G\left(\epsilon_{2}, c^{*}\right)-\frac{\gamma M_{4}}{M_{6}} \xi e^{\left(\rho^{*}-\epsilon_{2}\right) \xi} \leq 0 \quad$ for $\xi<\xi_{5}$.

Notice the fact that

$$
2 M_{4}^{2}(-\xi)^{3 / 2}\left(\xi-c^{*} \tau\right)^{2} e^{\rho^{*}\left(\xi-c^{*} \tau\right)}-2 M_{4} M_{6}(-\xi)^{3 / 2}\left(\xi-c^{*} \tau\right) e^{\epsilon_{2} \xi} \rightarrow 0 \quad \text { as } \xi \rightarrow-\infty,
$$

then there exists a sufficiently large constant $\left|\xi_{*}\right|$ with $\xi_{*}<0$ such that

$2 M_{4}^{2}(-\xi)^{3 / 2}\left(\xi-c^{*} \tau\right)^{2} e^{\rho^{*}\left(\xi-c^{*} \tau\right)}-2 M_{4} M_{6}(-\xi)^{3 / 2}\left(\xi-c^{*} \tau\right) e^{\epsilon_{2} \xi}<\frac{\left(c^{*} \tau\right)^{2}}{16} S_{1}$ and $1+\frac{c^{*} \tau}{\xi}>0$ for $\xi<\xi_{*}$. Define $\xi_{6}:=-M_{5}^{2} / M_{4}^{2}$ and take the constant $M_{5} \gg 1$ such that

$$
\xi_{6}<\xi_{4}, \quad \xi_{6}<\xi_{*} \quad \text { and } \quad S_{1}-\rho_{5}^{-1} e^{\rho_{5} \xi_{6}} \geq S_{1} / 2 .
$$

With the choices of above parameters, we introduce the following nonnegative continuous functions on $\mathbb{R}$.

$$
\begin{aligned}
& S_{+}^{*}(\xi):=S_{1}, \\
& S_{-}^{*}(\xi):= \begin{cases}S_{1}-\rho_{5}^{-1} e^{\rho_{5} \xi}, & \xi<\xi_{4}, \\
\sigma_{2} e^{-\frac{\beta}{c^{*}} \xi}, & \xi \geq \xi_{4},\end{cases} \\
& I_{+}^{*}(\xi):=\left\{\begin{array}{lll}
-M_{4} \xi e^{\rho^{*} \xi}, & \xi<\xi_{5}, \\
\left(R_{0}-1\right) S_{1}, & \xi \geq \xi_{5},
\end{array}\right. I_{-}^{*}(\xi):= \begin{cases}{\left[-M_{4} \xi-M_{5}(-\xi)^{1 / 2}\right] e^{\rho^{*} \xi},} & \xi<\xi_{6}, \\
0, & \xi \geq \xi_{6},\end{cases} \\
& R_{+}^{*}(\xi):=M_{6} e^{\epsilon_{2} \xi}, R_{-}^{*}(\xi):=0 .
\end{aligned}
$$

To illustrate that the parameters are admissible, we give Figures $5.1,5.2$ and 5.3 .
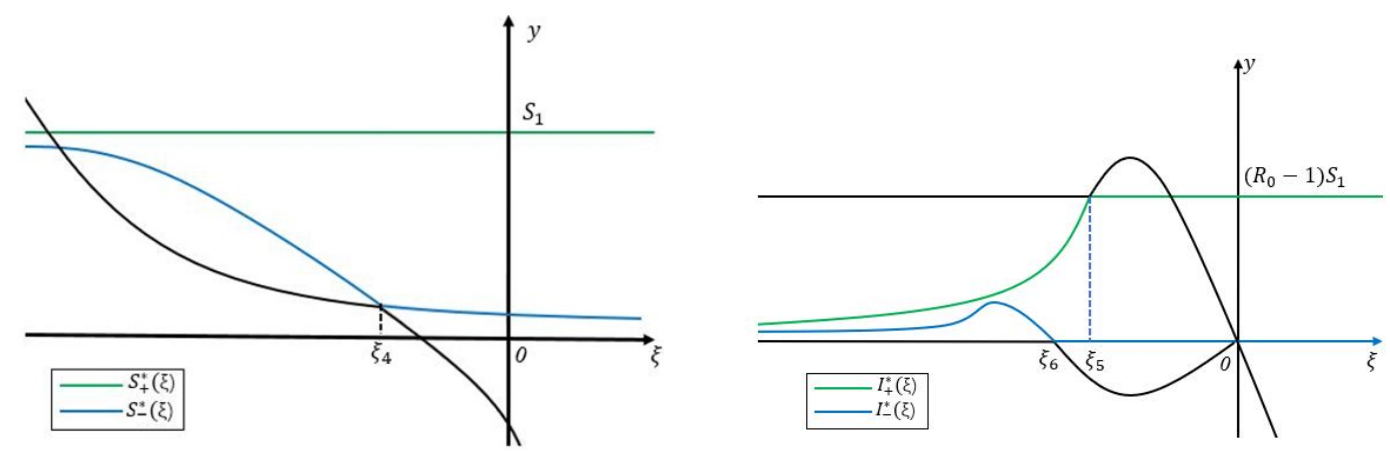

Figure 5.1: $S_{+}^{*}(\xi)$ and $S_{-}^{*}(\xi)$ when $R_{0}>1$ Figure 5.2: $I_{+}^{*}(\xi)$ and $I_{-}^{*}(\xi)$ when $R_{0}>1$ and $c=c^{*}$. and $c=c^{*}$. 


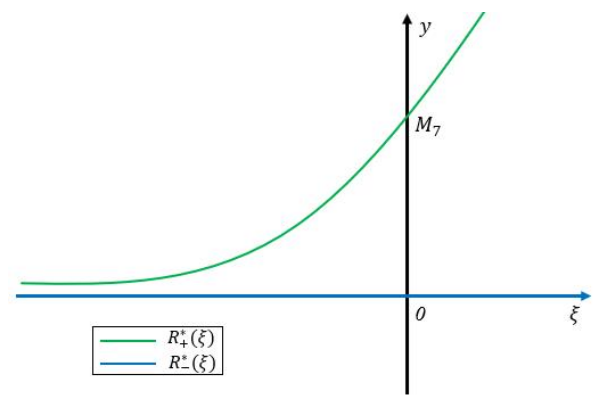

Figure 5.3: $R_{+}^{*}(\xi)$ and $R_{-}^{*}(\xi)$ when $R_{0}>1$ and $c=c^{*}$.

Lemma 5.1. The functions $S_{ \pm}^{*}(\xi), I_{ \pm}^{*}(\xi)$ and $R_{ \pm}^{*}(\xi)$ satisfy

$$
d_{s} D\left[S_{+}^{*}\right](\xi)-c^{*}\left(S_{+}^{*}\right)^{\prime}(\xi)-\frac{\beta S_{+}^{*}(\xi) I_{-}^{*}\left(\xi-c^{*} \tau\right)}{S_{+}^{*}(\xi)+I_{-}^{*}\left(\xi-c^{*} \tau\right)+R_{+}^{*}(\xi)} \leq 0, \quad \xi \in \mathbb{R},
$$

$$
d_{i} D\left[I_{+}^{*}\right](\xi)-c^{*}\left(I_{+}^{*}\right)^{\prime}(\xi)+\frac{\beta S_{+}^{*}(\xi) I_{+}^{*}\left(\xi-c^{*} \tau\right)}{S_{+}^{*}(\xi)+I_{+}^{*}\left(\xi-c^{*} \tau\right)+R_{-}^{*}(\xi)}-(\gamma+\delta) I_{+}^{*}(\xi) \leq 0, \quad \xi \neq \xi_{5},
$$

$$
d_{r} D\left[R_{+}^{*}\right](\xi)-c^{*}\left(R_{+}^{*}\right)^{\prime}(\xi)+\gamma I_{+}^{*}(\xi) \leq 0, \quad \xi \in \mathbb{R},
$$

$$
d_{s} D\left[S_{-}^{*}\right](\xi)-c^{*}\left(S_{-}^{*}\right)^{\prime}(\xi)-\frac{\beta S_{-}^{*}(\xi) I_{+}^{*}\left(\xi-c^{*} \tau\right)}{S_{-}^{*}(\xi)+I_{+}^{*}\left(\xi-c^{*} \tau\right)+R_{-}^{*}(\xi)} \geq 0, \quad \xi \neq \xi_{4},
$$

$$
\begin{gathered}
d_{i} D\left[I_{-}^{*}\right](\xi)-c^{*}\left(I_{-}^{*}\right)^{\prime}(\xi)+\frac{\beta S_{-}^{*}(\xi) I_{-}^{*}\left(\xi-c^{*} \tau\right)}{S_{-}^{*}(\xi)+I_{-}^{*}\left(\xi-c^{*} \tau\right)+R_{+}^{*}(\xi)}-(\gamma+\delta) I_{-}^{*}(\xi) \geq 0, \quad \xi \neq \xi_{6}, \\
d_{r} D\left[R_{-}^{*}\right](\xi)-c^{*}\left(R_{-}^{*}\right)^{\prime}(\xi)+\gamma I_{-}^{*}(\xi) \geq 0, \quad \xi \in \mathbb{R} .
\end{gathered}
$$

Proof. It is easy to verify that $(5.5)$ and $(5.10)$ hold under the definitions of $S_{+}^{*}(\xi), I_{-}^{*}(\xi)$ and $R_{ \pm}^{*}(\xi)$ on $\mathbb{R}$. The rest of the proof is devoted to proving inequalities $5.6-(5.9)$.

Proof of (5.6). When $\xi<\xi_{5}$, we get that $I_{+}^{*}(\xi)=-M_{4} \xi e^{\rho^{*} \xi}, I_{+}^{*}\left(\xi-c^{*} \tau\right)=-M_{4}(\xi-$ $\left.c^{*} \tau\right) e^{\rho^{*}\left(\xi-c^{*} \tau\right)}, I_{+}^{*}(\xi-1)=-M_{4}(\xi-1) e^{\rho^{*}(\xi-1)}, I_{+}^{*}(\xi+1) \leq-M_{4}(\xi+1) e^{\rho^{*}(\xi+1)}$ and $\left(I_{+}^{*}\right)^{\prime}(\xi)=-M_{4} e^{\rho^{*} \xi}\left(1+\rho^{*} \xi\right)$. Then by (2.1) and $(2.2)$, we compute that

$$
\begin{aligned}
& d_{i} D\left[I_{+}^{*}\right](\xi)-c^{*}\left(I_{+}^{*}\right)^{\prime}(\xi)+\frac{\beta S_{+}^{*}(\xi) I_{+}^{*}\left(\xi-c^{*} \tau\right)}{S_{+}^{*}(\xi)+I_{+}^{*}\left(\xi-c^{*} \tau\right)+R_{-}^{*}(\xi)}-(\gamma+\delta) I_{+}^{*}(\xi) \\
\leq & d_{i} D\left[I_{+}^{*}\right](\xi)-c^{*}\left(I_{+}^{*}\right)^{\prime}(\xi)+\beta I_{+}^{*}\left(\xi-c^{*} \tau\right)-(\gamma+\delta) I_{+}^{*}(\xi) \\
\leq & d_{i}\left[-M_{4}(\xi+1) e^{\rho^{*}(\xi+1)}-M_{4}(\xi-1) e^{\rho^{*}(\xi-1)}+2 M_{4} \xi e^{\rho^{*} \xi}\right] \\
& +c^{*} M_{4} e^{\rho^{*} \xi}\left(1+\rho^{*} \xi\right)-\beta M_{4}\left(\xi-c^{*} \tau\right) e^{\rho^{*}\left(\xi-c^{*} \tau\right)}+(\gamma+\delta) M_{4} \xi e^{\rho^{*} \xi} \\
= & -M_{4} \xi e^{\rho^{*} \xi} F\left(\rho^{*}, c^{*}\right)-M_{4} e^{\rho^{*} \xi} F_{\rho}\left(\rho^{*}, c^{*}\right)=0 .
\end{aligned}
$$

When $\xi>\xi_{5}$, we have that $I_{+}^{*}(\xi)=I_{+}^{*}(\xi+1)=\left(R_{0}-1\right) S_{1}, I_{+}^{*}\left(\xi-c^{*} \tau\right) \leq\left(R_{0}-1\right) S_{1}$, 
$I_{+}^{*}(\xi-1) \leq\left(R_{0}-1\right) S_{1}, S_{+}^{*}(\xi)=S_{1}$ and $R_{-}^{*}(\xi)=0$. Then we infer that

$$
\begin{aligned}
& d_{i} D\left[I_{+}^{*}\right](\xi)-c^{*}\left(I_{+}^{*}\right)^{\prime}(\xi)+\frac{\beta S_{+}^{*}(\xi) I_{+}^{*}\left(\xi-c^{*} \tau\right)}{S_{+}^{*}(\xi)+I_{+}^{*}\left(\xi-c^{*} \tau\right)+R_{-}^{*}(\xi)}-(\gamma+\delta) I_{+}^{*}(\xi) \\
\leq & \frac{\beta S_{1}\left(R_{0}-1\right) S_{1}}{S_{1}+\left(R_{0}-1\right) S_{1}}-(\gamma+\delta)\left(R_{0}-1\right) S_{1}=0 .
\end{aligned}
$$

Proof of $(5.7)$. By the expression of $I_{+}^{*}(\xi)$ and $R_{+}^{*}(\xi)$ on $\mathbb{R}$, we obtain from Lemma 2.3 and $(5.2)$ that

$$
\begin{aligned}
& d_{r} D\left[R_{+}^{*}\right](\xi)-c^{*}\left(R_{+}^{*}\right)^{\prime}(\xi)+\gamma I_{+}^{*}(\xi) \\
= & d_{r}\left[M_{6} e^{\epsilon_{2}(\xi+1)}+M_{6} e^{\epsilon_{2}(\xi-1)}-2 M_{6} e^{\epsilon_{2} \xi}\right]-c^{*} M_{6} \epsilon_{2} e^{\epsilon_{2} \xi}-\gamma M_{4} \xi e^{\rho^{*} \xi} \\
= & M_{6} e^{\epsilon_{2} \xi}\left[d_{r}\left(e^{\epsilon_{2}}+e^{-\epsilon_{2}}-2\right)-c^{*} \epsilon_{2}-\frac{\gamma M_{4} \xi e^{\left(\rho^{*}-\epsilon_{2}\right) \xi}}{M_{6}}\right] \\
= & M_{6} e^{\epsilon_{2} \xi}\left[-G\left(\epsilon_{2}, c^{*}\right)-\frac{\gamma M_{4} \xi e^{\left(\rho^{*}-\epsilon_{2}\right) \xi}}{M_{6}}\right] \leq 0 \quad \text { for } \xi<\xi_{5}
\end{aligned}
$$

and

$$
\begin{aligned}
& d_{r} D\left[R_{+}^{*}\right](\xi)-c^{*}\left(R_{+}^{*}\right)^{\prime}(\xi)+\gamma I_{+}^{*}(\xi) \\
= & d_{r}\left[M_{6} e^{\epsilon_{2}(\xi+1)}+M_{6} e^{\epsilon_{2}(\xi-1)}-2 M_{6} e^{\epsilon_{2} \xi}\right]-c^{*} M_{6} \epsilon_{2} e^{\epsilon_{2} \xi}+\gamma\left(R_{0}-1\right) S_{1} \\
= & M_{6} e^{\epsilon_{2} \xi}\left[d_{r}\left(e^{\epsilon_{2}}+e^{-\epsilon_{2}}-2\right)-c^{*} \epsilon_{2}+\frac{\gamma\left(R_{0}-1\right) S_{1} e^{-\epsilon_{2} \xi}}{M_{6}}\right] \\
= & M_{6} e^{\epsilon_{2} \xi}\left[-G\left(\epsilon_{2}, c^{*}\right)+\frac{\gamma\left(R_{0}-1\right) S_{1} e^{-\epsilon_{2} \xi_{5}}}{M_{6}}\right] \leq 0 \quad \text { for } \xi \geq \xi_{5} .
\end{aligned}
$$

Proof of 5.8). When $\xi<\xi_{4}, S_{-}^{*}(\xi)=S_{1}-\rho_{5}^{-1} e^{\rho_{5} \xi}, S_{-}^{*}(\xi-1)=S_{1}-\rho_{5}^{-1} e^{\rho_{5}(\xi-1)}$, $S_{-}^{*}(\xi+1) \geq S_{1}-\rho_{5}^{-1} e^{\rho_{5}(\xi+1)}, I_{+}^{*}\left(\xi-c^{*} \tau\right)=-M_{4}\left(\xi-c^{*} \tau\right) e^{\rho^{*}\left(\xi-c^{*} \tau\right)}$ and $R_{-}^{*}(\xi)=0$. Then it follows from (5.1) that

$$
\begin{aligned}
& d_{s} D\left[S_{-}^{*}\right](\xi)-c^{*}\left(S_{-}^{*}\right)^{\prime}(\xi)-\frac{\beta S_{-}^{*}(\xi) I_{+}^{*}\left(\xi-c^{*} \tau\right)}{S_{-}^{*}(\xi)+I_{+}^{*}\left(\xi-c^{*} \tau\right)+R_{-}^{*}(\xi)} \\
\geq & d_{s} D\left[S_{-}^{*}\right](\xi)-c^{*}\left(S_{-}^{*}\right)^{\prime}(\xi)-\beta I_{+}^{*}\left(\xi-c^{*} \tau\right) \\
\geq & d_{s}\left[2 \rho_{5}^{-1} e^{\rho_{5} \xi}-\rho_{5}^{-1} e^{\rho_{5}(\xi+1)}-\rho_{5}^{-1} e^{\rho_{5}(\xi-1)}\right]+c^{*} e^{\rho_{5} \xi}+\beta M_{4}\left(\xi-c^{*} \tau\right) e^{\rho^{*}\left(\xi-c^{*} \tau\right)} \\
= & e^{\rho_{5} \xi}\left[\rho_{5}^{-1} d_{s}\left(2-e^{\rho_{5}}-e^{-\rho_{5}}\right)+c^{*}+\beta M_{4}\left(\xi-c^{*} \tau\right) e^{\left(\rho^{*}-\rho_{5}\right) \xi-\rho^{*} c^{*} \tau}\right] \\
\geq & 0 \quad \text { for } \xi<\xi_{4} .
\end{aligned}
$$

When $\xi>\xi_{4}, S_{-}^{*}(\xi)=\sigma_{2} e^{-\frac{\beta}{c^{*}} \xi}, S_{-}^{*}(\xi+1)=\sigma_{2} e^{-\frac{\beta}{c^{*}}(\xi+1)}$ and $S_{-}^{*}(\xi-1) \geq \sigma_{2} e^{-\frac{\beta}{c^{*}}(\xi-1)}$. Then we have that

$$
d_{s} D\left[S_{-}^{*}\right](\xi)-c^{*}\left(S_{-}^{*}\right)^{\prime}(\xi)-\frac{\beta S_{-}^{*}(\xi) I_{+}^{*}\left(\xi-c^{*} \tau\right)}{S_{-}^{*}(\xi)+I_{+}^{*}\left(\xi-c^{*} \tau\right)+R_{-}^{*}(\xi)}
$$




$$
\begin{aligned}
& \geq d_{s} D\left[S_{-}^{*}\right](\xi)-c^{*}\left(S_{-}^{*}\right)^{\prime}(\xi)-\beta S_{-}^{*}(\xi) \\
& \geq d_{s}\left[\sigma_{2} e^{-\frac{\beta}{c^{*}}(\xi+1)}+\sigma_{2} e^{-\frac{\beta}{c^{*}}(\xi-1)}-2 \sigma_{2} e^{-\frac{\beta}{c^{*}} \xi}\right]+\beta \sigma_{2} e^{-\frac{\beta}{c^{*}} \xi}-\beta \sigma_{2} e^{-\frac{\beta}{c^{*}} \xi} \\
& =d_{s} \sigma_{2} e^{-\frac{\beta}{c^{*}} \xi}\left(e^{-\beta / c^{*}}+e^{\beta / c^{*}}-2\right) \\
& \geq 0 \quad \text { for } \xi>\xi_{4} .
\end{aligned}
$$

Proof of (5.9). By (5.4) we have for $\xi<\xi_{6}$ that

$$
\begin{aligned}
S_{-}^{*}(\xi) & =S_{1}-\rho_{5}^{-1} e^{\rho_{5} \xi} \geq S_{1} / 2 \\
I_{-}^{*}(\xi) & =\left[-M_{4} \xi-M_{5}(-\xi)^{1 / 2}\right] e^{\rho^{*} \xi} \\
\left(I_{-}^{*}\right)^{\prime}(\xi) & =\left[-M_{4}+\frac{1}{2} M_{5}(-\xi)^{-1 / 2}\right] e^{\rho^{*} \xi}+\left[-M_{4} \rho^{*} \xi-M_{5} \rho^{*}(-\xi)^{1 / 2}\right] e^{\rho^{*} \xi}, \\
I_{-}^{*}(\xi-1) & =\left[-M_{4}(\xi-1)-M_{5}(-\xi+1)^{1 / 2}\right] e^{\rho^{*}(\xi-1)}, \\
I_{-}^{*}\left(\xi-c^{*} \tau\right) & =\left[-M_{4}\left(\xi-c^{*} \tau\right)-M_{5}\left(-\xi+c^{*} \tau\right)^{1 / 2}\right] e^{\rho^{*}\left(\xi-c^{*} \tau\right)}, \\
I_{-}^{*}(\xi+1) & \geq\left[-M_{4}(\xi+1)-M_{5}(-\xi-1)^{1 / 2}\right] e^{\rho^{*}(\xi+1)} .
\end{aligned}
$$

Applying Taylor's formula, we deduce for $\xi<\xi_{6}$ that

$$
\begin{aligned}
(-\xi+1)^{1 / 2} & \leq(-\xi)^{1 / 2}+\frac{1}{2}(-\xi)^{-1 / 2} \\
(-\xi-1)^{1 / 2} & \leq(-\xi)^{1 / 2}-\frac{1}{2}(-\xi)^{-1 / 2} \\
\left(-\xi+c^{*} \tau\right)^{1 / 2} & \leq(-\xi)^{1 / 2}+\frac{c^{*} \tau}{2}(-\xi)^{-1 / 2}-\frac{\left(c^{*} \tau\right)^{2}}{8}(-\xi)^{-3 / 2}+\frac{\left(c^{*} \tau\right)^{3}}{16}(-\xi)^{-5 / 2} .
\end{aligned}
$$

Noting that $I_{-}^{*}\left(\xi-c^{*} \tau\right) \leq I_{+}^{*}\left(\xi-c^{*} \tau\right)=-M_{4}\left(\xi-c^{*} \tau\right) e^{\rho^{*}\left(\xi-c^{*} \tau\right)}$ for $\xi<\xi_{6}$, we get from (5.11) that

$$
\begin{aligned}
& -\beta I_{-}^{*}\left(\xi-c^{*} \tau\right)+\frac{\beta S_{-}^{*}(\xi) I_{-}\left(\xi-c^{*} \tau\right)}{S_{-}^{*}(\xi)+I_{-}^{*}\left(\xi-c^{*} \tau\right)+R_{+}^{*}(\xi)} \\
= & -\frac{\beta\left(I_{-}^{*}\right)^{2}\left(\xi-c^{*} \tau\right)+\beta I_{-}^{*}\left(\xi-c^{*} \tau\right) R_{+}^{*}(\xi)}{S_{-}^{*}(\xi)+I_{-}^{*}\left(\xi-c^{*} \tau\right)+R_{+}^{*}(\xi)} \geq-\frac{\beta\left(I_{-}^{*}\right)^{2}\left(\xi-c^{*} \tau\right)+\beta I_{-}^{*}\left(\xi-c^{*} \tau\right) R_{+}^{*}(\xi)}{S_{-}^{*}(\xi)} \\
\geq & -\frac{2 \beta}{S_{1}}\left[M_{4}^{2}\left(\xi-c^{*} \tau\right)^{2} e^{2 \rho^{*}\left(\xi-c^{*} \tau\right)}-M_{4} M_{6}\left(\xi-c^{*} \tau\right) e^{\left(\rho^{*}+\epsilon_{2}\right) \xi-\rho^{*} c^{*} \tau}\right] \quad \text { for } \xi<\xi_{6} .
\end{aligned}
$$

Using (5.3), (5.4), (5.11)-(5.13), (2.1) and $(2.2)$, we obtain for $\xi<\xi_{6}$ that

$$
\begin{aligned}
& d_{i} D\left[I_{-}^{*}\right](\xi)-c^{*}\left(I_{-}^{*}\right)^{\prime}(\xi)+\frac{\beta S_{-}^{*}(\xi) I_{-}^{*}\left(\xi-c^{*} \tau\right)}{S_{-}^{*}(\xi)+I_{-}^{*}\left(\xi-c^{*} \tau\right)+R_{+}^{*}(\xi)}-(\gamma+\delta) I_{-}^{*}(\xi) \\
= & d_{i} D\left[I_{-}^{*}\right](\xi)-c^{*}\left(I_{-}^{*}\right)^{\prime}(\xi)+\beta I_{-}^{*}\left(\xi-c^{*} \tau\right)-(\gamma+\delta) I_{-}^{*}(\xi)-\beta I_{-}^{*}\left(\xi-c^{*} \tau\right) \\
& +\frac{\beta S_{-}^{*}(\xi) I_{-}^{*}\left(\xi-c^{*} \tau\right)}{S_{-}^{*}(\xi)+I_{-}^{*}\left(\xi-c^{*} \tau\right)+R_{+}^{*}(\xi)}
\end{aligned}
$$




$$
\begin{aligned}
& \geq d_{i}\left\{\left[-M_{4}(\xi+1)-M_{5}(-\xi-1)^{1 / 2}\right] e^{\rho^{*}(\xi+1)}+\left[-M_{4}(\xi-1)-M_{5}(-\xi+1)^{1 / 2}\right] e^{\rho^{*}(\xi-1)}\right. \\
& \left.-2\left[-M_{4} \xi-M_{5}(-\xi)^{1 / 2}\right] e^{\rho^{*} \xi}\right\} \\
& -c^{*}\left\{\left[-M_{4}+\frac{1}{2} M_{5}(-\xi)^{-1 / 2}\right] e^{\rho^{*} \xi}+\left[-M_{4} \rho^{*} \xi-M_{5} \rho^{*}(-\xi)^{1 / 2}\right] e^{\rho^{*} \xi}\right\} \\
& +\beta\left[-M_{4}\left(\xi-c^{*} \tau\right)-M_{5}\left(-\xi+c^{*} \tau\right)^{1 / 2}\right] e^{\rho^{*}\left(\xi-c^{*} \tau\right)}-(\gamma+\delta)\left[-M_{4} \xi-M_{5}(-\xi)^{1 / 2}\right] e^{\rho^{*} \xi} \\
& -\frac{2 \beta}{S_{1}}\left[M_{4}^{2}\left(\xi-c^{*} \tau\right)^{2} e^{2 \rho^{*}\left(\xi-c^{*} \tau\right)}-M_{4} M_{6}\left(\xi-c^{*} \tau\right) e^{\left(\rho^{*}+\epsilon_{2}\right) \xi-\rho^{*} c^{*} \tau}\right] \\
& \geq d_{i}\left\{\left[-M_{4}(\xi+1)-M_{5}\left((-\xi)^{1 / 2}-\frac{1}{2}(-\xi)^{-1 / 2}\right)\right] e^{\rho^{*}(\xi+1)}\right. \\
& \left.+\left[-M_{4}(\xi-1)-M_{5}\left((-\xi)^{1 / 2}+\frac{1}{2}(-\xi)^{-1 / 2}\right)\right] e^{\rho^{*}(\xi-1)}-2\left[-M_{4} \xi-M_{5}(-\xi)^{1 / 2}\right] e^{\rho^{*} \xi}\right\} \\
& -c^{*}\left[-M_{4}+\frac{1}{2} M_{5}(-\xi)^{-1 / 2}\right] e^{\rho^{*} \xi}-c^{*} \rho^{*}\left[-M_{4} \xi-M_{5}(-\xi)^{1 / 2}\right] e^{\rho^{*} \xi}-\beta M_{4}\left(\xi-c^{*} \tau\right) e^{\rho^{*}\left(\xi-c^{*} \tau\right)} \\
& -\beta M_{5}\left[(-\xi)^{1 / 2}+\frac{c^{*} \tau}{2}(-\xi)^{-1 / 2}-\frac{\left(c^{*} \tau\right)^{2}}{8}(-\xi)^{-3 / 2}+\frac{\left(c^{*} \tau\right)^{3}}{16}(-\xi)^{-5 / 2}\right] e^{\rho^{*}\left(\xi-c^{*} \tau\right)} \\
& -(\gamma+\delta)\left[-M_{4} \xi-M_{5}(-\xi)^{1 / 2}\right] e^{\rho^{*} \xi} \\
& -\frac{2 \beta}{S_{1}}\left[M_{4}^{2}\left(\xi-c^{*} \tau\right)^{2} e^{2 \rho^{*}\left(\xi-c^{*} \tau\right)}-M_{4} M_{6}\left(\xi-c^{*} \tau\right) e^{\left(\rho^{*}+\epsilon_{2}\right) \xi-\rho^{*} c^{*} \tau}\right] \\
& =\left[-M_{4} \xi-M_{5}(-\xi)^{1 / 2}\right] e^{\rho^{*} \xi} F\left(\rho^{*}, c^{*}\right)+\left[-M_{4}+\frac{1}{2} M_{5}(-\xi)^{-1 / 2}\right] e^{\rho^{*} \xi} F_{\rho}\left(\rho^{*}, c^{*}\right) \\
& +\beta M_{5}\left[\frac{\left(c^{*} \tau\right)^{2}}{8}(-\xi)^{-3 / 2}-\frac{\left(c^{*} \tau\right)^{3}}{16}(-\xi)^{-5 / 2}\right] e^{\rho^{*}\left(\xi-c^{*} \tau\right)} \\
& -\frac{2 \beta}{S_{1}}\left[M_{4}^{2}\left(\xi-c^{*} \tau\right)^{2} e^{2 \rho^{*}\left(\xi-c^{*} \tau\right)}-M_{4} M_{6}\left(\xi-c^{*} \tau\right) e^{\left(\rho^{*}+\epsilon_{2}\right) \xi-\rho^{*} c^{*} \tau}\right] \\
& =\beta M_{5}(-\xi)^{-3 / 2} e^{\rho^{*}\left(\xi-c^{*} \tau\right)} \frac{\left(c^{*} \tau\right)^{2}}{16}\left(1+\frac{c^{*} \tau}{\xi}\right) \\
& +\frac{\beta}{S_{1}}(-\xi)^{-3 / 2} e^{\rho^{*}\left(\xi-c^{*} \tau\right)} \\
& \times\left[\frac{\left(c^{*} \tau\right)^{2}}{16} M_{5} S_{1}-2 M_{4}^{2}(-\xi)^{3 / 2}\left(\xi-c^{*} \tau\right)^{2} e^{\rho^{*}\left(\xi-c^{*} \tau\right)}+2 M_{4} M_{6}(-\xi)^{3 / 2}\left(\xi-c^{*} \tau\right) e^{\epsilon_{2} \xi}\right]
\end{aligned}
$$

$\geq 0$.

When $\xi>\xi_{6}, I_{-}^{*}(\xi)=0$ and inequality 5.9 follows trivially. The claim of this lemma is shown.

Now we state the existence result of critical traveling wave solution for (1.1).

Theorem 5.2. For a given constant $S_{1}>0$, if $R_{0}>1$ and $c=c^{*}$, then system (1.1) has a traveling wave solution $(S, I, R)(\xi)$ satisfying the following assertions.

(i) $0<S(\xi)<S_{1}, 0<I(\xi)<\left(R_{0}-1\right) S_{1}$ and $R(\xi)>0$ for $\xi \in \mathbb{R}$.

(ii) $(S, I, R)(-\infty)=\left(S_{1}, 0,0\right)$. If $\xi \rightarrow-\infty$, then $I(\xi)=O\left(-\xi e^{\rho^{*} \xi}\right)$. 
(iii) $I(+\infty)=0, S(+\infty):=S_{2}$ exists and $S_{2}<S_{1}$.

(iv) $(\gamma+\delta) \int_{\mathbb{R}} I(\xi) d \xi=\beta \int_{\mathbb{R}} \frac{S(\xi) I\left(\xi-c^{*} \tau\right)}{S(\xi)+I\left(\xi-c^{*} \tau\right)+R(\xi)} d \xi=c^{*}\left(S_{1}-S_{2}\right)$.

(v) If $\lim \sup _{\xi \rightarrow+\infty} R(\xi)<+\infty$, then $R(+\infty)=\gamma\left(S_{1}-S_{2}\right) /(\gamma+\delta)$ and $S^{\prime}(\xi), I^{\prime}(\xi), R^{\prime}(\xi)$ $\rightarrow 0$ as $\xi \rightarrow \pm \infty$.

(vi) $\frac{\partial c^{*}}{\partial d_{i}}>0, \frac{\partial c^{*}}{\partial \beta}>0$ and $\frac{\partial c^{*}}{\partial \tau}<0$.

Proof. Applying the functions $S_{ \pm}^{*}(\xi), I_{ \pm}^{*}(\xi)$ and $R_{ \pm}^{*}(\xi)$ defined at the beginning of this section and the analogous discussions in Section 4, we can obtain system (1.1) admits a critical traveling wave solution $(S, I, R)(\xi)$ satisfying $(\mathrm{i})-(\mathrm{v})$. In the following, we shall prove (vi) of this theorem. From Lemma 2.2, we can compute that

$$
\begin{gathered}
F_{c^{*}}\left(\rho^{*}, c^{*}\right)=-\rho^{*}-\beta \rho^{*} \tau e^{-\rho^{*} c^{*} \tau}<0, \quad F_{d_{i}}\left(\rho^{*}, c^{*}\right)=e^{\rho^{*}}+e^{-\rho^{*}}-2>0, \\
F_{\beta}\left(\rho^{*}, c^{*}\right)=e^{-\rho^{*} c^{*} \tau}>0 \quad \text { and } \quad F_{\tau}\left(\rho^{*}, c^{*}\right)=-\beta \rho^{*} c^{*} e^{-\rho^{*} c^{*} \tau}<0,
\end{gathered}
$$

which together with derivative rule for implicit functions implies that

$$
\frac{\partial c^{*}}{\partial d_{i}}>0, \quad \frac{\partial c^{*}}{\partial \beta}>0 \quad \text { and } \quad \frac{\partial c^{*}}{\partial \tau}<0 .
$$

The proof of this theorem is completed.

Remark 5.3. Theorems 3.1, 4.5 and 5.2 mainly reveal the sufficient conditions of existence and nonexistence of traveling wave solutions for (1.1), and the characterization of their minimal speed. One can observe that the obtained traveling waves include the pulse-type ( $I$-component) and front-type ( $S$-component and $R$-component) traveling waves. Notice that $I(-\infty)=0$ for both $c>c^{*}$ and $c=c^{*}$, while the exact decay rates of $I(-\infty)=0$ are distinct for these cases. The limit value $I(+\infty)=0$ implies that the infected individuals will disappear after a long time.

Remark 5.4. Inequalities in Theorem 5.2(vi) reflect that the geographical movement of the infected individuals and the interaction between infected individuals and susceptible individuals can accelerate the speed of propagation of the epidemic, while the time delay can slow down the speed of transmission of the epidemic.

By Theorems 4.5 and 5.2 , we can further obtain some properties concerning the functions $S(\xi), I(\xi)$ and $R(\xi)$.

Proposition 5.5. Let $(S, I, R)(\xi)$ be a nontrivial positive traveling wave solutions of (1.1) for each $c \geq c^{*}$. Then for $j=1,2,3$, the functions $e^{k_{j} \xi} U_{j}(\xi)$ are strictly increasing and $U_{j}(\xi \pm 1) / U_{j}(\xi)$ are uniformly bounded on $\mathbb{R}$, where $k_{1}=\left(2 d_{s}+\beta\right) / c, k_{2}=\left(2 d_{i}+\gamma+\delta\right) / c$, $k_{3}=2 d_{r} / c, U_{1}(\xi)=S(\xi), U_{2}(\xi)=I(\xi)$ and $U_{3}(\xi)=R(\xi)$. 
Proof. Here we only prove the corresponding results for $R(\xi)$ since one can use the similar arguments to deduce the remainder results. By the third equation in $(1.2)$, we have

$$
\begin{aligned}
{\left[e^{\frac{2 d_{r}}{c} \xi} R(\xi)\right]^{\prime} } & =e^{\frac{2 d_{r}}{c} \xi} R^{\prime}(\xi)+\frac{2 d_{r}}{c} e^{\frac{2 d_{r}}{c} \xi} R(\xi) \\
& =\frac{d_{r}}{c} e^{\frac{2 d_{r}}{c} \xi}[R(\xi+1)+R(\xi-1)]+\frac{\gamma}{c} e^{\frac{2 d_{r}}{c} \xi} I(\xi)>0
\end{aligned}
$$

for $\xi \in \mathbb{R}$, which implies that the function $e^{\frac{2 d_{r}}{c} \xi} R(\xi)$ is strictly increasing on $\mathbb{R}$. Thus we obtain that

$$
e^{\frac{2 d_{r}}{c} \xi} R(\xi)>e^{\frac{2 d_{r}}{c}(\xi-1)} R(\xi-1) \quad \text { for } \xi \in \mathbb{R}
$$

which is equivalent to

$$
\frac{R(\xi-1)}{R(\xi)}<e^{2 d_{r} / c} \quad \text { for } \xi \in \mathbb{R}
$$

Observing (5.14) gives

$$
\left[e^{\frac{2 d_{r}}{c} \xi} R(\xi)\right]^{\prime}>\frac{d_{r}}{c} e^{\frac{2 d_{r}}{c} \xi} R(\xi+1) \quad \text { for } \xi \in \mathbb{R} .
$$

Integrating 5.15 over $[\xi, \xi+1]$ and using the monotonicity of $e^{\frac{2 d_{r}}{c} \xi} R(\xi)$, we obtain

$$
\begin{aligned}
e^{\frac{2 d_{r}}{c}(\xi+1)} R(\xi+1)-e^{\frac{2 d_{r}}{c} \xi} R(\xi) & \geq \frac{d_{r}}{c} \int_{\xi}^{\xi+1} e^{\frac{2 d_{r}}{c} \eta} R(\eta+1) d \eta \\
& >\frac{d_{r}}{c} e^{\frac{2 d_{r}}{c} \xi} R(\xi+1) \quad \text { for } \xi \in \mathbb{R},
\end{aligned}
$$

that is,

$$
R(\xi+1)>\left[R(\xi)+\frac{d_{r}}{c} R(\xi+1)\right] e^{-2 d_{r} / c} \quad \text { for } \xi \in \mathbb{R}
$$

Inserting (5.16) into (5.15) yields

$$
\begin{aligned}
{\left[e^{\frac{2 d_{r}}{c} \xi} R(\xi)\right]^{\prime} } & >\frac{d_{r}}{c} e^{\frac{2 d_{r}}{c} \xi}\left[R(\xi)+\frac{d_{r}}{c} R(\xi+1)\right] e^{-2 d_{r} / c} \\
& >\frac{d_{r}^{2}}{c^{2}} e^{-4 d_{r} / c} e^{\frac{2 d_{r}}{c}(\xi+1)} R(\xi+1) \quad \text { for } \xi \in \mathbb{R} .
\end{aligned}
$$

Integrating (5.17) over $[\xi-1 / 2, \xi]$ gives

$$
\begin{aligned}
e^{\frac{2 d_{r}}{c} \xi} R(\xi) & \geq e^{\frac{2 d_{r}}{c}(\xi-1 / 2)} R(\xi-1 / 2)+\frac{d_{r}^{2}}{c^{2}} e^{-4 d_{r} / c} \int_{\xi-1 / 2}^{\xi} e^{\frac{2 d_{r}}{c}(\eta+1)} R(\eta+1) d \eta \\
& >\frac{d_{r}^{2}}{2 c^{2}} e^{-4 d_{r} / c} e^{\frac{2 d_{r}}{c}(\xi+1 / 2)} R(\xi+1 / 2) \quad \text { for } \xi \in \mathbb{R},
\end{aligned}
$$

where we have used the monotonicity of $e^{\frac{2 d_{r}}{c} \xi} R(\xi)$. Then it follows from (5.18) that

$$
\frac{R(\xi+1 / 2)}{R(\xi)}<\frac{2 c^{2}}{d_{r}^{2}} e^{3 d_{r} / c} \quad \text { for } \xi \in \mathbb{R}
$$


which implies that

$$
\frac{R(\xi+1)}{R(\xi)}=\frac{R(\xi+1)}{R(\xi+1 / 2)} \cdot \frac{R(\xi+1 / 2)}{R(\xi)}<\frac{4 c^{4}}{d_{r}^{4}} e^{6 d_{r} / c} \quad \text { for } \xi \in \mathbb{R} .
$$

The proof is completed.

\section{Acknowledgments}

The authors are very grateful to the anonymous referee for his/her constructive comments and suggestions, which have helped to improve our original manuscript greatly. This work was supported by National Natural Science Foundation of China [grant numbers 12001241 \& 11731014], China Postdoctoral Science Foundation [grant number 2018M642173], Basic Research Program of Jiangsu Province [grant number BK20200885] and Jiangsu Key Lab for Numerical Simulation of Large Scale Complex Systems [grant number 202006].

\section{References}

[1] S. Ai and R. Albashaireh, Traveling waves in spatial SIRS models, J. Dynam. Differential Equations 26 (2014), no. 1, 143-164.

[2] F. Andreu-Vaillo, J. M. Mazón, J. D. Rossi and J. J. Toledo-Melero, Nonlocal Diffusion Problems, Mathematical Surveys and Monographs 165, American Mathematical Society, Providence, RI, 2010.

[3] F. Brauer, P. van den Driessche and J. Wu, Mathematical Epidemiology, Lecture Notes in Mathematics 1945, Springer-Verlag, New York, 2008.

[4] X. Chen and J.-S. Guo, Uniqueness and existence of traveling waves for discrete quasilinear monostable dynamics, Math. Ann. 326 (2003), no. 1, 123-146.

[5] Y.-Y. Chen, J.-S. Guo and F. Hamel, Traveling waves for a lattice dynamical system arising in a diffusive endemic model, Nonlinearity 30 (2017), no. 6, 2334-2359.

[6] Y. Cheng, D. Lu, J. Zhou and J. Wei, Existence of traveling wave solutions with critical speed in a delayed diffusive epidemic model, Adv. Difference Equ. 2019 (2019), Paper No. 494, 21 pp.

[7] A. Ducrot, M. Langlais and P. Magal, Qualitative analysis and travelling wave solutions for the SI model with vertical transmission, Commun. Pure Appl. Anal. 11 (2012), no. 1, 97-113. 
[8] A. Ducrot and P. Magal, Travelling wave solutions for an infection-age structured epidemic model with external supplies, Nonlinearity 24 (2011), no. 10, 2891-2911.

[9] P. Fife, Some nonclassical trends in parabolic and parabolic-like evolutions, in: Trends in Nonlinear Analysis, 153-191, Springer, Berlin, 2003.

[10] S.-C. Fu, Traveling waves for a diffusive SIR model with delay, J. Math. Anal. Appl. 435 (2016), no. 1, 20-37.

[11] S.-C. Fu, J.-S. Guo and C.-C. Wu, Traveling wave solutions for a discrete diffusive epidemic model, J. Nonlinear Convex Anal. 17 (2016), no. 9, 1739-1751.

[12] J. He and J.-C. Tsai, Traveling waves in the Kermack-McKendrick epidemic model with latent period, Z. Angew. Math. Phys. 70 (2019), no. 1, Paper No. 27, 22 pp.

[13] H. W. Hethcote, The mathematics of infectious diseases, SIAM Rev. 42 (2000), no. 4, 599-653.

[14] Y. Hosono and B. Ilyas, Traveling waves for a simple diffusive epidemic model, Math. Models Methods Appl. Sci. 5 (1995), no. 7, 935-966.

[15] V. Hutson, S. Martinez, K. Mischaikow and G. T. Vickers, The evolution of dispersal, J. Math. Biol. 47 (2003), no. 6, 483-517.

[16] C.-Y. Kao, Y. Lou and W. Shen, Random dispersal vs. non-local dispersal, Discrete Contin. Dyn. Syst. 26 (2010), no. 2, 551-596.

[17] _ Evolution of mixed dispersal in periodic environments, Discrete Contin. Dyn. Syst. Ser. B 17 (2012), no. 6, 2047-2072.

[18] Y. Li, W.-T. Li and F.-Y. Yang, Traveling waves for a nonlocal dispersal SIR model with delay and external supplies, Appl. Math. Comput. 247 (2014), 723-740.

[19] Y. Li, W.-T. Li and G.-B. Zhang, Stability and uniqueness of traveling waves of a non-local dispersal SIR epidemic model, Dyn. Partial Differ. Equ. 14 (2017), no. 2, 87-123.

[20] H. Wang and X.-S. Wang, Traveling wave phenomena in a Kermack-McKendrick SIR model, J. Dynam. Differential Equations 28 (2016), no. 1, 143-166.

[21] J.-B. Wang, W.-T. Li and F.-Y. Yang, Traveling waves in a nonlocal dispersal SIR model with nonlocal delayed transmission, Commun. Nonlinear Sci. Numer. Simul. 27 (2015), no. 1-3, 136-152. 
[22] X.-S. Wang, H. Wang and J. Wu, Traveling waves of diffusive predator-prey systems: disease outbreak propagation, Discrete Contin. Dyn. Syst. 32 (2012), no. 9, 3303-3324.

[23] Z.-C. Wang and J. Wu, Travelling waves of a diffusive Kermack-McKendrick epidemic model with non-local delayed transmission, Proc. R. Soc. Lond. Ser. A Math. Phys. Eng. Sci. 466 (2010), no. 2113, 237-261.

[24] J. Wei, Asymptotic boundary and nonexistence of traveling waves in a discrete diffusive epidemic model, J. Difference Equ. Appl. 26 (2020), no. 2, 163-170.

[25] J. Wei, J. Zhou, W. Chen, Z. Zhen and L. Tian, Traveling waves in a nonlocal dispersal epidemic model with spatio-temporal delay, Commun. Pure Appl. Anal. 19 (2020), no. 5, 2853-2886.

[26] J. Wei, J. Zhou, Z. Zhen and L. Tian, Super-critical and critical traveling waves in a two-component lattice dynamical model with discrete delay, Appl. Math. Comput. 363 (2019), 124621, 15 pp.

[27] _ Super-critical and critical traveling waves in a three-component delayed disease system with mixed diffusion, J. Comput. Appl. Math. 367 (2020), 112451, 23 pp.

[28] _ Time periodic traveling waves in a three-component non-autonomous and reaction-diffusion epidemic model, Accepted in International Journal of Mathematics, 2020.

[29] D. V. Widder, The Laplace Transform, Princeton Mathematical Series 6, Princeton University Press, Princeton, N.J., 1941.

[30] C.-C. Wu, Existence of traveling waves with the critical speed for a discrete diffusive epidemic model, J. Differential Equations 262 (2017), no. 1, 272-282.

[31] J. Wu and X. Zou, Traveling wave fronts of reaction-diffusion systems with delay, J. Dynam. Differential Equations 13 (2001), no. 3, 651-687.

[32] Z. Xu and C. Ai, Traveling waves in a diffusive influenza epidemic model with vaccination, Appl. Math. Model. 40 (2016), no. 15-16, 7265-7280.

[33] F.-Y. Yang and W.-T. Li, Traveling waves in a nonlocal dispersal SIR model with critical wave speed, J. Math. Anal. Appl. 458 (2018), no. 2, 1131-1146.

[34] F.-Y. Yang, W.-T. Li and Z.-C. Wang, Traveling waves in a nonlocal dispersal SIR epidemic model, Nonlinear Anal. Real World Appl. 23 (2015), 129-147. 
[35] F.-Y. Yang, Y. Li, W.-T. Li and Z.-C. Wang, Traveling waves in a nonlocal dispersal Kermack-McKendrick epidemic model, Discrete Contin. Dyn. Syst. Ser. B 18 (2013), no. 7, 1969-1993.

[36] Q. Zhang and S.-L. Wu, Wave propagation of a discrete SIR epidemic model with a saturated incidence rate, Int. J. Biomath. 12 (2019), no. 3, 1950029, 18 pp.

[37] T. Zhang and W. Wang, Existence of traveling wave solutions for influenza model with treatment, J. Math. Anal. Appl. 419 (2014), no. 1, 469-495.

[38] L. Zhao, Z.-C. Wang and S. Ruan, Traveling wave solutions in a two-group epidemic model with latent period, Nonlinearity 30 (2017), no. 4, 1287-1325.

[39] _ Traveling wave solutions in a two-group SIR epidemic model with constant recruitment, J. Math. Biol. 77 (2018), no. 6-7, 1871-1915.

[40] Z. Zhen, J. Wei, L. Tian, J. Zhou and W. Chen, Wave propagation in a diffusive SIR epidemic model with spatiotemporal delay, Math. Methods Appl. Sci. 41 (2018), no. 16, 7074-7098.

[41] Z. Zhen, J. Wei, J. Zhou and L. Tian, Wave propagation in a nonlocal diffusion epidemic model with nonlocal delayed effects, Appl. Math. Comput. 339 (2018), 1537.

[42] J. Zhou, L. Song and J. Wei, Mixed types of waves in a discrete diffusive epidemic model with nonlinear incidence and time delay, J. Differential Equations 268 (2020), no. 8, 4491-4524.

[43] J. Zhou, L. Song, J. Wei and H. Xu, Critical traveling waves in a diffusive disease model, J. Math. Anal. Appl. 476 (2019), no. 2, 522-538.

[44] J. Zhou, J. Xu, J. Wei and H. Xu, Existence and non-existence of traveling wave solutions for a nonlocal dispersal SIR epidemic model with nonlinear incidence rate, Nonlinear Anal. Real World Appl. 41 (2018), 204-231.

Jingdong Wei, Zaili Zhen, Jiangbo Zhou and Lixin Tian

School of Mathematical Sciences, Jiangsu University, Zhenjiang, Jiangsu 212013, China E-mail addresses: wjd19871022@126.com, zhenzaili@ujs.edu.cn, ujszjb@126.com, tianlx@ujs.edu.cn 